\title{
Measuring the Heating and Cooling of the Interstellar Medium at High Redshift: PAH and [C II] Observations of the Same Star-forming Galaxies at $z \sim 2$
}

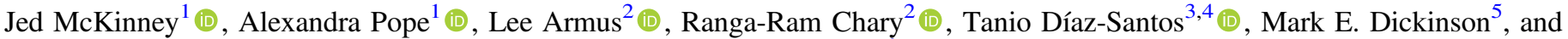 \\ Allison Kirkpatrick ${ }^{6}$ (1) \\ ${ }^{1}$ Department of Astronomy, University of Massachusetts, Amherst, MA 01003, USA; jhmckinney@umass.edu \\ ${ }^{2}$ Infrared Processing and Analysis Center, MC 314-6, Caltech, 1200 E. California Boulevard, Pasadena, CA 91125, USA \\ ${ }^{3}$ Núcleo de Astronomía de la Facultad de Ingeniería y Ciencias, Universidad Diego Portales, Av. Ejército Libertador 441, Santiago, Chile \\ ${ }^{4}$ Chinese Academy of Sciences South America Center for Astronomy, National Astronomical Observatories, CAS, Beijing 100101, China \\ ${ }_{5}^{5}$ National Optical Astronomy Observatory, 950 North Cherry Avenue, Tucson, AZ 85719, USA \\ ${ }^{6}$ Department of Physics \& Astronomy, University of Kansas, Lawrence, KS 66045, USA \\ Received 2019 November 26; revised 2020 February 12; accepted 2020 February 17; published 2020 April 3
}

\begin{abstract}
Star formation depends critically on cooling mechanisms in the interstellar medium (ISM); however, thermal properties of gas in galaxies at the peak epoch of star formation $(z \sim 2)$ remain poorly understood. A limiting factor in understanding the multiphase ISM is the lack of multiple tracers detected in the same galaxies, such as Polycyclic Aromatic Hydrocarbon (PAH) emission, a tracer of a critical photoelectric heating mechanism in interstellar gas, and [C II] $158 \mu \mathrm{m}$ fine-structure emission, a principal coolant. We present ALMA Band 9 observations targeting [C II] in six $z \sim 2$ star-forming galaxies with strong Spitzer IRS detections of PAH emission. All six galaxies are detected in dust continuum and marginally resolved. We compare the properties of $\mathrm{PAH}$ and $[\mathrm{C} \mathrm{II}]$ emission, and constrain their relationship as a function of total infrared luminosity $\left(L_{\mathrm{IR}}\right)$ and IR surface density. [C II] emission is detected in one galaxy at high signal-to-noise $(34 \sigma)$, and we place a secure upper limit on a second source. The rest of our sample are not detected in [C II] likely due to redshift uncertainties and narrow ALMA bandpass windows. Our results are consistent with the deficit in $[\mathrm{C} \mathrm{II}] / L_{\mathrm{IR}}$ and $\mathrm{PAH} / L_{\mathrm{IR}}$ observed in the literature. However, the ratio of [C II] to PAH emission at $z \sim 2$ is possibly much lower than what is observed in nearby dusty star-forming galaxies. This could be the result of enhanced cooling via [O I] at high- $z$, hotter gas and dust temperatures, and/or a reduction in the photoelectric efficiency, in which the coupling between interstellar radiation and gas heating is diminished.
\end{abstract}

Unified Astronomy Thesaurus concepts: Starburst galaxies (1570); Galaxies (573); Galaxy evolution (594); Interstellar medium (847); High-redshift galaxies (734); Ultraluminous infrared galaxies (1735)

\section{Introduction}

Ten billion years ago $(z \sim 2)$, the star-formation-rate density of the universe peaked and individual galaxies were forming more stars than at any other time in cosmic history (e.g., Lilly et al. 1996; Madau et al. 1996; Chary \& Elbaz 2001; Madau \& Dickinson 2014). Enhanced star formation was promoted by gas resupply through cold-mode accretion onto galaxies (e.g., Kereš et al. 2005, 2009; Genzel et al. 2008; Tacconi et al. 2010), accompanied by a change in the efficiency of star formation (e.g., Tacconi et al. 2010, 2013, 2018; Genzel et al. 2015; Scoville et al. 2017; Liu et al. 2019), which suggests evolution in the heating and cooling mechanisms of interstellar gas.

The internal transfer of thermal energy is critical for any physical system. Photoelectrons ejected from polycyclic aromatic hydrocarbons (PAHs) are thought to be the most important, albeit inefficient, mechanism for converting stellar radiation to thermal energy in and around sites of active star formation (Watson \& Salpeter 1972; Bakes \& Tielens 1994; Helou et al. 2001). PAH molecules are complex grains comprised mostly of $\mathrm{C}$ and $\mathrm{H}$, and they are common in photodissociation regions (PDRs) where gas densities of $n \sim 10^{3}-10^{6} \mathrm{~cm}^{-3}$ are illuminated by far-UV stellar radiation fields (Tielens \& Hollenbach 1985). Once excited by stellar photons, PAHs emit vibrational lines between 5 and $15 \mu \mathrm{m}$ that can contain as much as $\sim 20 \%$ of total IR emission $\left(L_{\mathrm{IR}}\right.$, 8-1000 $\mu \mathrm{m}$; Sajina et al. 2007; Smith et al. 2007; Pope et al. 2008; Dale et al. 2009). Therefore, mid-IR PAH features are direct probes of photoelectric heating in dense PDRs and a key diagnostic of the interstellar medium (ISM).

The energy injected into the ISM by photoelectrons is radiated away in the infrared (IR). Far-IR fine-structure emission lines such as [C II] at $158 \mu \mathrm{m}$ and [O I] at $63 \mu \mathrm{m}$ can contain $0.1 \%-1 \%$ of $L_{\mathrm{IR}}$ (Tielens \& Hollenbach 1985; Stacey et al. 2010; Díaz-Santos et al. 2013; Brisbin et al. 2015; Ibar et al. 2015). [C II] in particular is emerging as a powerful but complicated diagnostic of the ISM because it comes from different regions in a galaxy. With a critical density of $n_{\text {crit }} \sim 3 \times 10^{3}-6 \times 10^{3} \mathrm{~cm}^{-3}$ at $\sim 100 \mathrm{~K},[\mathrm{C} \mathrm{II}]$ is collisionally excited by $\mathrm{H}$ and $\mathrm{H}_{2}$ in PDRs, as well as by warm electrons at $8000 \mathrm{~K}$ (Goldsmith et al. 2012). Ancillary observations of [N II] $205 \mu \mathrm{m}$ emission constrain the fraction of [C II] emission originating from PDRs (e.g., Croxall et al. 2012), which is greater for lower metallicities (Croxall et al. 2017; Cormier et al. 2019) and approaches unity in warm and compact, dusty, star-forming regions (Sutter et al. 2019). Thus, [C II] can be used to trace PDR cooling in warm, compact environments, a critical physical process in atomic gas for star formation to occur.

PDR densities are much greater than the critical density of [C II] with its primary collisional partners $\mathrm{H}$ and $\mathrm{H}_{2}$ $\left(n_{\text {crit }, \mathrm{H}}=3000 \mathrm{~cm}^{-3}, n_{\text {crit, } \mathrm{H}_{2}}=6100 \mathrm{~cm}^{-3}\right)$, both of which are heated by photoelectrons from PAH grains (Tielens \& Hollenbach 1985; Wolfire et al. 1990; Kaufman et al. 1999; Malhotra et al. 2001; Goldsmith et al. 2012). Thus, a correlation 
between [C II] and PAH emission is likely if both lines originate from the same PDR regions. Indeed, Helou et al. (2001) found the ratio of [C II] emission over integrated 5-10 $\mu \mathrm{m}$ flux in starforming galaxies to be independent of far-IR color, which strongly favors a co-spatial origin.

Pope et al. (2013) report a deficit of $6.2 \mu \mathrm{m}$ PAH emission at higher $L_{\mathrm{IR}}$ in (ultra) luminous IR galaxies (LIRGs: $\log L_{\mathrm{IR}} / L_{\odot}=11-12$, ULIRGs: $\left.\log L_{\mathrm{IR}} / L_{\odot}>12\right)$ and submillimeter $(\mathrm{mm})$ galaxies, a feature also observed for [C II] emission in similar galaxy populations. Indeed, the luminosity ratio of [C II] to $L_{\mathrm{IR}}$ decreases at higher $L_{\mathrm{IR}}$ in local and high- $z$ galaxies. ${ }^{7}$ In low- $z$ (U)LIRGs, Díaz-Santos et al. (2013, 2017) find $L_{[\mathrm{C} \text { II] }} / L_{\mathrm{IR}}$ empirically anticorrelates with average dust temperatures and IR-luminosity surface densities, suggesting that either harder and more intense radiation fields lower the $L_{[\mathrm{C} \text { II] }} / L_{\mathrm{IR}}$ ratio, or larger dust grains out-compete PAHs for ionizing photons, starving the gas. Smith et al. (2017) find the star-formation-rate surface density to be a primary factor driving the [C II]-deficit, reconciling nearby resolved measurements and high- $z$ galaxies with a relation that spans over six orders of magnitude. At $z \sim 3$, Rybak et al. (2019) find evidence for thermal saturation of $\mathrm{C}^{+}$as the primary driver of the deficit (see also Muñoz \& Oh 2016). Other potential contributors to the [C II]-deficit include positive PAH grain charging where fewer photoelectrons are available to collisionally excite [C II] (e.g., Helou et al. 2001), density effects (e.g., Smith et al. 2017), and/or [C II] self-absorption, although the latter scenario requires unusually large gas column densities in PDRs and is unlikely (Luhman et al. 1998; Malhotra et al. 2001).

Regardless of its origin, the [C II]-deficit implies that one of the most important cooling lines for star formation falls off in luminosity at higher $L_{\mathrm{IR}}$, or equivalently, higher star formation rate (SFR; Kennicutt 1998). This implies a change in one or all of the following: the photoelectric heating efficiency of the ISM, far-UV radiation field strength and hardness, gas density and PDR geometry (Smith et al. 2017). Furthermore, galaxies have higher SFR per unit stellar mass at earlier times than they do locally (e.g., Madau \& Dickinson 2014), suggesting that ISM conditions evolve as a function of redshift and SFR (Scoville et al. 2017; Tacconi et al. 2018; Liu et al. 2019). Indeed, Stacey et al. (2010) found that the [C II]-deficit is pushed to higher $L_{\mathrm{IR}}$ at higher redshifts; however, Zanella et al. (2018) did not observe this offset in a sample of main-sequence galaxies at $z \sim 2$. In either case, all galaxies may follow the same $L_{[\mathrm{C} \text { II] }} / L_{\mathrm{IR}}$ trend as a function of $L_{\mathrm{IR}}$ normalized by molecular gas mass (Stacey et al. 2010; Graciá-Carpio et al. 2011). Thus, the gas cooling properties and stellar radiation field strengths in local and $z>1$ star-forming galaxies could be comparable for a given star formation efficiency $\left(\mathrm{SFE} \equiv \mathrm{SFR} / M_{\mathrm{H}_{2}}\right.$ ). If this is the case, high- $z$ star formation could be a scaled up version of star formation today with comparable ISM conditions, and therefore, similar mid- and far-IR PDR line ratios.

In this paper, we combine new observations using the Atacama Large Millimeter/submillimeter Array (ALMA) to investigate the properties of ISM heating and cooling in $z \sim 2$ star-forming galaxies through combined observations of [C II] and PAH emission. With archival Spitzer Infrared Spectrograph

\footnotetext{
7 Exempli gratia, Malhotra et al. (1997, 2001), Luhman et al. (1998, 2003), Helou et al. (2001), Díaz-Santos et al. (2013, 2014, 2017), Stacey et al. (2010), Magdis et al. (2014), Rigopoulou et al. (2014), Brisbin et al. (2015), Ibar et al. (2015), Zanella et al. (2018), and Rybak et al. (2019).
}

(IRS) spectra, we can identify pure star-forming galaxies to study the properties of $z \sim 2$ PDRs and star formation without concern for feedback from an active galactic nucleus (AGN). Using ratios of [C II] to PAH emission, we investigate the photoelectric efficiency in PDRs near the peak in the universe's star formation rate density, a critical epoch for galaxy evolution during which most of the stellar mass in the present day universe was assembled (e.g., Madau \& Dickinson 2014). We investigate the evolution in [C II]/PAH emission with redshift, and comment on the technical aspects of synergistic surveys combining ALMA and mid-IR spectrographs, with applications to the James Webb Space Telescope Mid-Infrared Instrument (JWST/MIRI).

The paper is organized as follows: In Section 2, we present the galaxy sample, selection criterion, and observations including novel and archival data. Our analysis techniques and emission line measurements are described in Section 3. We present our results in Section 4 and discuss their implications in Section 5. Section 6 summarizes our conclusions. Throughout this work, we assume a Salpeter IMF and adopt a $\Lambda$ CDM cosmology with $\Omega_{m}=0.3, \Omega_{\Lambda}=0.7$, and $H_{0}=70 \mathrm{~km} \mathrm{~s}^{-1} \mathrm{Mpc}^{-1}$.

\section{Sample and Observations}

\subsection{Sample Selection}

We have assembled a sample of six IR-luminous galaxies $\left(\log L_{\mathrm{IR}} / L_{\odot}>12\right)$ at $z=1.7-2$ with extensive coverage from restframe ultraviolet to sub-mm wavelengths, selected primarily by the presence of luminous PAH features in the mid-IR and little to no underlying power-law continuum. These systems are dominated by star formation: an AGN would heat dust to high temperatures and emits warm blackbody emission at mid-IR wavelengths that we do not detect (e.g., Laurent et al. 2000; Sturm et al. 2000; Tran et al. 2001; Sajina et al. 2007). Our sample comes from a larger multiwavelength parent catalog described in Kirkpatrick et al. (2015). To summarize, multiwavelength data was collected for 343 (U)LIRGs between $z=0.3-2.8$ in the Great Observatories Origins Deep Survey North/South (GOODS-N/S), Extended Chandra Deep Field Survey (ECDFS), and the Spitzer Extragalactic First Look Survey fields. The primary target selection criterion was the presence of mid-IR spectroscopy from Spitzer IRS. For more details on the parent sample selection method, we refer readers to Section 2.1 of Kirkpatrick et al. (2015).

With our ALMA cycle 5 program targeting [C II] emission at $z \sim 2$, we observed six star-forming galaxies between $z=1.7-1.9$ from the Kirkpatrick et al. (2015) sample with $L_{\mathrm{PAH}, 6.2} / L_{\mathrm{IR}}>0.004$ and $\log L_{\mathrm{IR}} / L_{\odot}>12$. These galaxies all have little to no evidence of AGN contamination to the mid-IR spectrum $\left(f_{\mathrm{AGN}, \mathrm{MIR}}\right)$, based on IRS spectral decomposition, and as evidenced by their $6.2 \mu \mathrm{m}$ PAH equivalent widths $\mathrm{EW}_{6.2}>0.5 \mu \mathrm{m}$, which is the threshold established in nearby (U)LIRGs for star formation dominated systems (Stierwalt et al. 2014). The selection of sources based on strong PAH features in high IR-luminosity galaxies has been shown in the literature to be a robust way for selecting galaxies with minimal AGN contamination (e.g., Houck et al. 2005; Yan et al. 2005; Brandl et al. 2006; Armus et al. 2007; Sajina et al. 2007; Smith et al. 2007; Pope et al. 2008; Veilleux et al. 2009; Kirkpatrick et al. 2012).

Configuring the ALMA Band 9 Local Oscillator to efficiently target [C II] over the redshift range spanned by our sample was a challenging factor in the design of our experiment. Efficient 
programs capable of observing multiple targets with minimal baseband tunings are optimal for taking advantage of limited high-frequency ALMA observing time. To maximize sample size while minimizing overhead, we manually configured each spectral window within the Band 9 constraints to cover [C II] in multiple galaxies in a given ALMA Science Goal.

Most of the galaxies in our sample have robust stellar masses constrained by deep HST and Spitzer photometry. Galaxies in our sample are high mass, $\log M_{*} / M_{\odot}=10.6-11$, and dusty, as evidenced by Spitzer and Hershel photometry. Figure 1 shows the star-forming main sequence (MS) of galaxies at $z \sim 2$ taken from Speagle et al. (2014). Our sample lies above the $z \sim 2$ SFMS, with $\log \triangle$ SFMS (the observed SFR over the MS SFR for the same stellar mass) between 0.6 and 1 dex for most galaxies in our sample and as high as $\sim 1.2$ dex in GS IRS20, well within the starburst domain. Table 1 summarizes global properties for galaxies in our sample.

\subsection{Multiwavelength Observations}

Our sources are in ECDFS and were selected to have mid-IR spectroscopy from the Spitzer IRS (Fadda et al. 2010; Kirkpatrick et al. 2012, 2015). A full description of IRS observations, data reduction, and sample selection can be found in Pope et al. (2008) and Kirkpatrick et al. (2012). The extracted spectra are shown in Figure 2 with simple fits to the mid-IR emission that we use to calculate $f_{\mathrm{AGN} \text {,MIR }}$; more sophisticated model fits are employed to measure PAH line luminosities (see Appendix A). In addition to Spitzer IRS spectra, photometry from Herschel (PACS and SPIRE), and Spitzer (IRAC and MIPS) is available for all targets (Table 2; see Kirkpatrick et al. 2015 for details).

ECDFS includes the GOODS-S field, which was covered by the Cosmic Assembly Near-IR Deep Extragalactic Legacy Survey (CANDELS; Grogin et al. 2011; Koekemoer et al. 2011), providing deep WFC3/IR imaging for five out of six galaxies in our sample. We downloaded the $H_{160}$ and $Z_{850 l p}$ field maps and correct for the known systematic astrometric offset of 0 ". 08 in RA and 0". 26 in DEC relative to ALMA's astrometry in GOODS-S (Elbaz et al. 2018). Thumbnail images for our sample are shown in Figure 3. We also matched our galaxies to visual morphological classifications presented in Kartaltepe et al. (2015) to assess the incidence of mergers in the sample.

\subsection{ALMA Observations and Data Processing}

We carried out ALMA Band 9 observations of our targets during Cycle 5 (PI A. Pope, Project ID: 2017.1.01347.S) targeting [C II] emission at restframe $157.74 \mu \mathrm{m}$. For the range of redshifts in our sample, [C II] is redshifted to an observed frequency of $653.36-686.38 \mathrm{GHz}$. We estimated integration times necessary to detect the [C II] line at $10 \sigma$ for galaxies in our sample by assuming a conservative $L_{[\mathrm{C} \mathrm{II}} / L_{\mathrm{IR}}$ ratio of 0.002 and [C II] line width of $300 \mathrm{~km} \mathrm{~s}^{-1}$, characteristic of existing [C II] detections $z \sim 2$ galaxies prior to our observations (Stacey et al. 2010). The minimum predicted [C II] flux for all galaxies in the sample was $15 \mathrm{Jy} \mathrm{km} \mathrm{s}^{-1}$, which we used to set the integration time for each observation by requiring a $>10 \sigma$ line detection, or equivalently, a sensitivity of $5 \mathrm{mJy}$ over $300 \mathrm{~km} \mathrm{~s}^{-1}$ bandwidth.

To avoid resolving out $[\mathrm{C}$ II] emission at $z \sim 2$, we requested an angular resolution of $\sim 0$." 5 . The observations took place in 2018 July in ALMA configuration C43-1, which has an angular

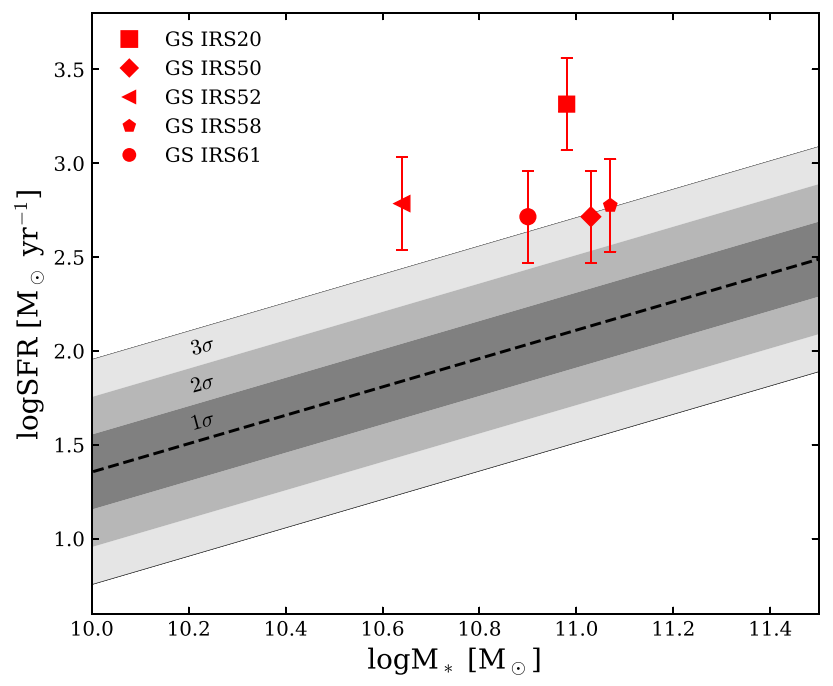

Figure 1. The $z \sim 2$ star-forming main sequence of Speagle et al. (2014; dashed black line) assumes the same (Salpeter) IMF used in our calculations. We shade $1 \sigma, 2 \sigma$, and $3 \sigma$ intrinsic scatter about the main sequence in gray. Galaxies from our sample are shown in red, excluding GS IRS46, which does not have a robust stellar mass estimate and is not detected in [C II] with ALMA. We only detect [C II] emission in GS IRS20 (red square), the starburst galaxy located $>5 \sigma$ above the main sequence.

resolution of 0 ". 52 at $650 \mathrm{GHz}$ and maximum recoverable scale of 4 ." 4 , corresponding to $36.5 \mathrm{kpc}$ at $z=2$. The expected radii of sub-mm and $H S T H_{160}$ emission in $z \sim 2$ star-forming galaxies is $<8 \mathrm{kpc}$ (e.g., Figure 3, Calistro Rivera et al. 2018; Zanella et al. 2018; Lang et al. 2019), so it is unlikely that our observations are missing flux on large scales due to interferometric spatial filtering. Six galaxies in our proposal were observed for $\sim 18$ minutes on-source, achieving a target sensitivity of $5 \mathrm{mJy}$ over $300 \mathrm{~km} \mathrm{~s}^{-1}$ bandwidth at a native resolution of $31.250 \mathrm{MHz}\left(13.6 \mathrm{~km} \mathrm{~s}^{-1}\right)$, which was later rebinned to lower spectral resolutions.

The data were reduced using the standard ALMA pipeline in CASA v5.1.1-5 (McMullin et al. 2007). We first imaged the data using tclean with Briggs weighting in continuum-mode, iteratively adjusting the robust parameter $R$ to maximize the ratio of peak continuum emission to map rms. We extracted peak and integrated continuum flux densities through elliptical apertures, which were set by fitting a 2D Gaussian function to the bright continuum emission in each observation. We detect continuum emission at representative frequencies of $652-699 \mathrm{GHz}$ in all of our targets at a signal-to-noise ratio $(\mathrm{S} / \mathrm{N})$ between 7.5 and 17.8. After verifying the presence of an underlying continuum, we created a linear continuum model in the $u v$-plane, taking care to mask out high-amplitude visibilities that could correspond to potential line emission. Next, we continuum-subtracted the ALMA cubes in the $u v$-plane and imaged the spectral windows with tclean and Briggs weighting using $R=0.5$. Final continuum measurements, ALMA beam characteristics, and spectral line statistics are given in Table 3.

\section{Analysis}

\section{1. [C II] Detection in GS IRS20}

Whereas the dust continuum is clearly detected with ALMA for all six galaxies (red contours in Figure 3), [C II] $158 \mu$ m emission 
Table 1

Sample Summary

\begin{tabular}{|c|c|c|c|c|c|c|c|c|c|}
\hline Target & $\begin{array}{l}\text { R.A. } \\
\text { (J2000) }\end{array}$ & $\begin{array}{l}\text { Decl. } \\
(\mathrm{J} 2000)\end{array}$ & $z_{\text {IRS }}$ & $\begin{array}{c}\log L_{\mathrm{IR}} \\
\left(L_{\odot}\right)\end{array}$ & $\begin{array}{c}\log L_{6.2 \mu \mathrm{m}} \\
\left(L_{\odot}\right)\end{array}$ & $\begin{array}{c}\log L_{11.3 \mu \mathrm{m}} \\
\left(L_{\odot}\right)\end{array}$ & $\begin{array}{c}\log M_{*}^{\mathrm{a}} \\
\left(M_{\odot}\right)\end{array}$ & $\begin{array}{c}\mathrm{SFR}_{\mathrm{IR}} \\
\left(M_{\odot} \mathrm{yr}^{-1}\right)\end{array}$ & $f_{\mathrm{AGN}, \mathrm{MIR}}{ }^{\mathrm{b}}$ \\
\hline GS IRS20 & $03: 32: 47.58$ & $-27: 44: 52.0$ & $1.923_{-0.030}^{+0.030}$ & $13.06 \pm 0.12$ & $9.99_{-0.12}^{+0.12}$ & $10.11_{-0.10}^{+0.10}$ & 10.98 & 717 & 0.2 \\
\hline GS IRS50 & $03: 32: 31.52$ & $-27: 48: 53.0$ & $1.900_{-0.041}^{+0.081}$ & $12.46 \pm 0.15$ & $10.17_{-0.09}^{+0.09}$ & $9.66_{-0.33}^{+0.33}$ & 11.03 & 184 & 0.0 \\
\hline GS IRS52 & $03: 32: 12.52$ & $-27: 43: 06.0$ & $1.824_{-0.020}^{+0.018}$ & $12.53 \pm 0.29$ & $9.91_{-0.12}^{+0.12}$ & $10.10_{-0.13}^{+0.13}$ & 10.64 & 232 & 0.0 \\
\hline GS IRS58 & $03: 32: 40.24$ & $-27: 49: 49.0$ & $1.890_{-0.042}^{+0.017}$ & $12.52 \pm 0.17$ & $9.91_{-0.10}^{+0.10}$ & $9.96_{-0.25}^{+0.25}$ & 11.07 & 207 & 0.0 \\
\hline
\end{tabular}

Notes. When calculating $M_{*}$ and SFR $\mathrm{IR}$, we assume a Salpeter IMF and $\mathrm{SFR}_{\mathrm{IR}} \approx 1.8 \times 10^{-10} L_{\mathrm{IR}}$ (Kennicutt 1998). We assume a systematic error of $10 \%$ for $L_{\mathrm{IR}}$ and include this in the quoted $1 \sigma$ uncertainty, all calculations, and on all figures. Appendix A describes our procedure for calculating PAH line luminosities and $z_{\text {IRS }}$. ${ }^{\text {a }}$ See Kirkpatrick et al. (2012) for details on stellar mass calculations.

b $f_{\text {AGN,MIR }}$ is the integrated AGN power-law emission divided by the total mid-IR IRS flux and is calculated using the mid-IR decomposition technique of Pope et al. (2008) and Kirkpatrick et al. (2015). We re-fit this template-based model using MCMC and calculate $f_{\mathrm{AGN}, \mathrm{MIR}}$ at each step in the Markov chain. Tabulated values for $f_{\mathrm{AGN}, \mathrm{MIR}}$ correspond to the mean of this distribution. Given the data in hand, $f_{\mathrm{AGN}, \mathrm{MIR}}$ can be measured with an accuracy of \pm 0.1 (e.g., Pope et al. 2008 ; Kirkpatrick et al. 2015).

c The $11.3 \mu \mathrm{m}$ PAH feature is redshifted out of GS IRS46's IRS spectrum.

${ }^{\mathrm{d}}$ GS IRS46 is outside of GOODS-S and CANDELS, preventing the calculation of a stellar mass with comparable methods to the rest of the sample for which deeper data is available.

is clearly detected in one of six galaxies in the sample, GS IRS20, at an observed frequency of $650.2505 \mathrm{GHz}$. This corresponds to a redshift of $z_{[\mathrm{C} \mathrm{II}}=1.9239 \pm 0.0002$, in excellent agreement with the PAH-derived redshift: $z_{[\mathrm{C} \text { II] }}-z_{\mathrm{PAH}}=0.001$.

We imaged the cube in $30 \mathrm{~km} \mathrm{~s}^{-1}$ bins, and extracted a spectrum through an elliptical aperture with FWHM and centroid taken from a 2D Gaussian fit to continuum emission. Figure 4 shows the detection of [C II] in GS IRS20's ALMA Band 9 spectrum. Gaps in spectral coverage are the result of limitations when configuring ALMA's spectral windows. We integrated the line over the frequency range where emission rose above the continuum level and measured a flux density of $S_{[\mathrm{C} \mathrm{II]}} \Delta v=$ $9.95 \pm 0.07 \mathrm{Jy} \mathrm{km} \mathrm{s}^{-1}$ at an $\mathrm{S} / \mathrm{N}$ of 34.3 and line velocity width of $\sim 330 \mathrm{~km} \mathrm{~s}^{-1}$. Next, we calculated the [C II] line luminosity $L_{\text {[C II] }}$ in solar units following Carilli \& Walter (2013):

$$
L_{[\mathrm{C} \mathrm{II}]}=1.04 \times 10^{-3} \times S_{[\mathrm{C} \text { II }} \Delta v D_{L}^{2} \nu_{\mathrm{obs}}\left[L_{\odot}\right]
$$

where $D_{L}$ is the luminosity distance in $\mathrm{Mpc}$, and $\nu_{\mathrm{obs}}$ is the observed frequency of the line in GHz. From the Band 9 spectrum, we calculate $\log L_{[\mathrm{C} \mathrm{II}]} / L_{\odot}=9.169 \pm 0.003$ in GS IRS20, the highest $\mathrm{S} / \mathrm{N}$ detection of [C II] emission in a $z \sim 2$ galaxy to date. From a collapsed ALMA data cube containing only line emission, we find that [C II] in GS IRS20 is marginally resolved with a spatial FWHM of 0 "!56, corresponding to $\approx 4.7 \mathrm{kpc}$ at $z=1.9239$.

\section{2. [C II] Line Searches and Upper Limits}

No [C II] emission lines were obvious in the ALMA cubes of GS IRS46, GS IRS50, GS IRS52, GS IRS58, and GS IRS61. To search for marginally detected emission lines, we used a circular aperture with radius 0.15 to extract a $50 \mathrm{~km} \mathrm{~s}^{-1}$ spectrum centered on the source's dust continuum position. Next, we extracted additional spectra through the same circular apertures offset by $0 . " 5$ from the source's center at various angles, as optical light, dust continuum, and [C II] emission can be spatially offset from one another in high-redshift ULIRGs (e.g., Calistro Rivera et al. 2018; Zanella et al. 2018). From the set of extracted spectra, we searched each spectral window for the presence of three channels greater than $2 \times$ the local rms. No marginally significant line emission was discovered in this manner or in stacks of the extracted spectra.

Given that $83 \%$ of our observations yielded non-detections, and no data was discarded because of poor atmospheric transmission, two explanations are possible. Either the observations were not deep enough to detect [C II] and an upper limit may be placed on $L_{[\mathrm{C} \text { II] }}$; or, the line was missed by our ALMA bandpass tunings. To determine which observations can yield a secure upper limit on $L_{[\mathrm{C} \text { II] }}$, we calculate $p(l \mid \mathrm{ALMA}, \Delta z)$ : the probability that our ALMA tunings covered the [C II] line given all redshift uncertainties and the comparatively narrow bandpass widths. The technique adopted for calculating $p(l \mid$ ALMA, $\Delta z)$ is described in detail in Appendix B. In summary, we integrate redshift probability distribution functions in spectral domains with ALMA coverage. We found this detailed analysis to be crucial for interpreting the data. Table 3 includes values of $p(l \mid$ ALMA, $\Delta z)$ for all targets.

Among the non-detections, only GS IRS61 has $p(l \mid$ ALMA, $\Delta z)>90 \%$. For this galaxy, we first calculate the rms over a spectrum at $50 \mathrm{~km} \mathrm{~s}^{-1}$ resolution $\left(\mathrm{rms}_{50}\right)$, extracted from an aperture centered on the dust continuum. Then, we calculate the $3 \sigma$ upper limit on the line luminosity using Equation (1) with $S_{\text {[C II] }} \Delta v=3 \Delta v\left(\sqrt{6} \mathrm{rms}_{50}\right)$, assuming $\Delta v=300 \mathrm{~km} \mathrm{~s}^{-1}$ as is observed in GS IRS20. Our upper limits for GS IRS61 on $L_{\text {[C II] }}$ are summarized in Table 3 and could be a factor $1.8(0.25$ dex $)$ larger than what is reported if we assume a more extreme $\Delta v=600 \mathrm{~km} \mathrm{~s}^{-1}$, greater than the noise-weighted average of $\sim 430 \mathrm{~km} \mathrm{~s}^{-1}$ as observed in [C II]-emitters at $z \sim 2-3$ (e.g., the sample of Gullberg et al. 2015).

\subsection{Morphology}

In all of our observations, dust continuum emission is marginally resolved: the major and minor axes of 2D Gaussian fits to dust emission are equal to 0 ". $5-2^{\prime \prime}$, slightly greater than the ALMA beam in all cases. We use these size measurements to calculate $R_{\text {eff, } 160 \text {, the radius containing } 50 \% \text { of the total }}$ continuum flux at the effective restframe wavelengths 

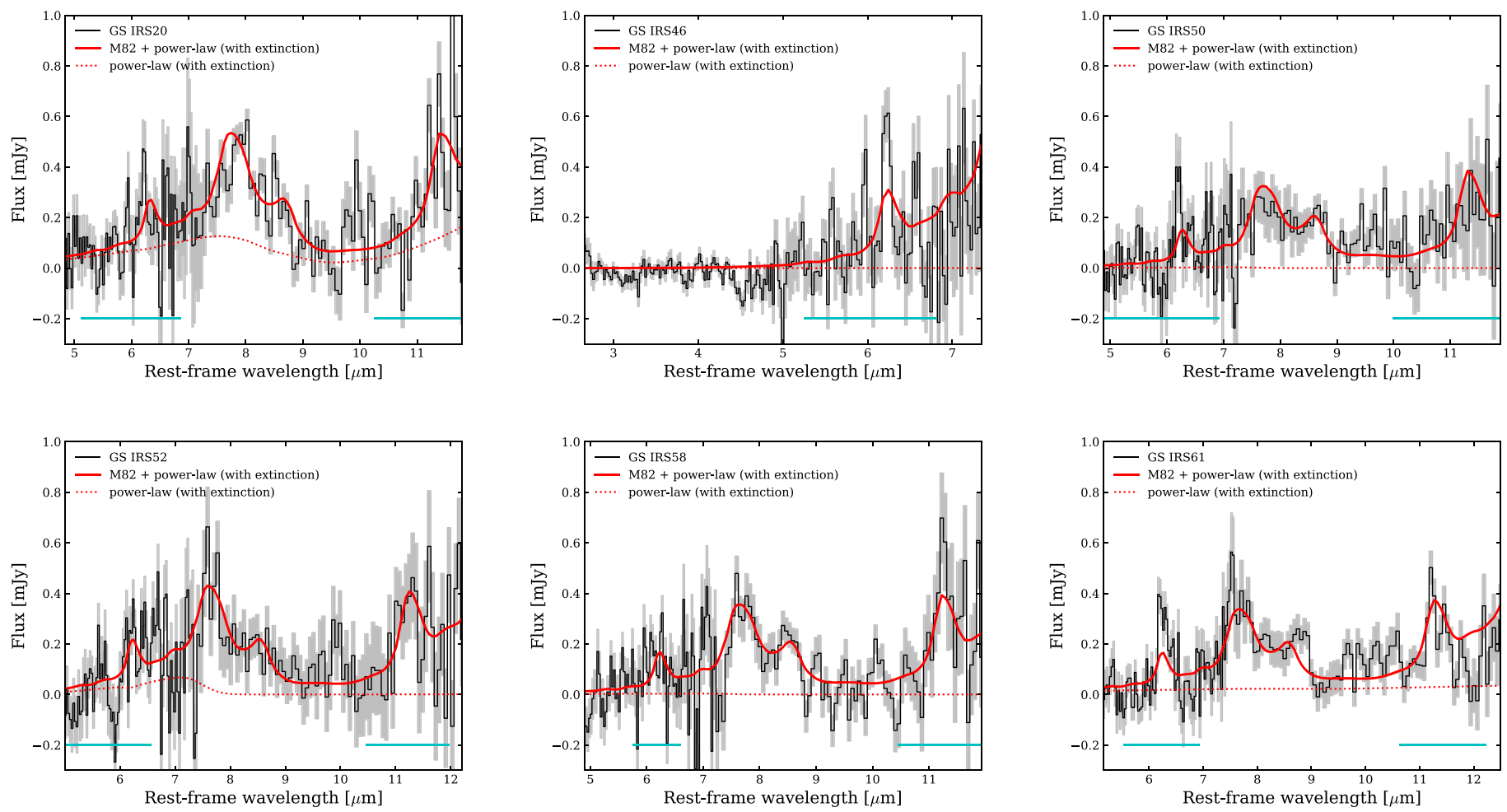

Figure 2. Spitzer IRS spectra for the six targets observed with ALMA. The IRS spectra are shown in black, with uncertainties shaded in gray. The red solid line corresponds to best-fit empirical M82 templates on top of a power-law continuum component (red dotted line). The simple fit is used to quantify the AGN fraction in the mid-IR, and we employ a more sophisticated model to measure individual line luminosities and redshifts (see Appendix A). The horizontal cyan lines show the regions where we fit Lorentzian profiles to the 6.2 and $11.3 \mu \mathrm{m}$ PAH features.

(approximately $160 \mu \mathrm{m}$ ) of our observations. Table 3 includes values of $R_{\text {eff, } 160 \text {, which we use to calculate IR surface }}$ densities. Given that the extent of the dust continuum is marginally greater than the ALMA beam in all cases, our measurements of $R_{\text {eff, } 160}$ may be thought of as upper limits.

Our ability to distinguish substructure in the ALMA maps is limited; however, extended $H_{160}$ emission in the $H S T$ thumbnails of GS IRS20, GS IRS50, and GS IRS58 suggests disturbed, perhaps merger-driven, morphologies in some cases. We matched our sources to the morphological classification catalog of Kartaltepe et al. (2015) to determine the incidence of mergers in our sample. Each of our targets had the maximum 68 classifications per galaxy. GS IRS20 is considered to be a merger by $80 \%$ of classifiers, and irregular by $100 \%$, consistent with its position $>5 \sigma$ above the $z=2$ galaxy main sequence (Figure 1), and the presence of faint extended $H_{160}$ emission to the northeast, reminiscent of a tidally disrupted stellar population. Dust continuum and [C II] emission in GS IRS20 are co-spatial and coincide with the $H_{160}$ maximum.

The rest of the sample was not classified as mergers, and GS IRS61 is classified as a spheroid by the full set of classifiers. The spatial extent of the $H_{160}$-band and dust continuum in GS IRS61 is $\sim 5 \mathrm{kpc}$ (FWHM), making this galaxy extremely compact. GS IRS46 is offset from the HST/ACS z-band map by 0 " 70 after correcting for the astrometry offset between $H S T$ and ALMA (see Section 2.2). This corresponds to $\sim 6 \mathrm{kpc}$ physical offset between the detected stellar light and dust continuum emission in this galaxy. Given the uncertainty introduced by this offset, we do not report a stellar mass or show optical data points for this dusty galaxy.

\subsection{Comparison Samples}

Since we have selected our $z \sim 2$ sample to include only starformation-dominated systems, we emphasize literature comparison samples with comparable selections $\left(\mathrm{EW}_{6.2 \mu \mathrm{m}}>0.5 \mu \mathrm{m}\right.$, Stierwalt et al. 2014). For comparison with local (U)LIRGs, we use mid- and far-IR spectral line measurements from DíazSantos et al. (2013, 2014, 2017) and Stierwalt et al. (2014) for galaxies in the Great Observatories All Sky LIRG Survey (GOALS; Armus et al. 2009). To contextualize PAH and [C II] line luminosities at lower $L_{\mathrm{IR}}$, we also compare our data to the intermediate-z $5 \mathrm{mJy}$ Unbiased Spitzer Extragalactic Survey (5MUSES; Wu et al. 2010), nearby galaxies from Sargsyan et al. (2014), Magdis et al. (2014), and Ibar et al. (2015). To characterize the landscape of [C II] observations at $z \sim 2$, we also compare our [C II] measurements to $z \sim 2-3$ galaxies with data from ALMA, APEX, or Herschel FTS (Ivison et al. 2010; Valtchanov et al. 2011; Gullberg et al. 2015; Schaerer et al. 2015; Hashimoto et al. 2018; Zanella et al. 2018; Rybak et al. 2019). Prior observations of both PAH and [C II] in the same galaxy at $z \sim 2$ are limited to a handful of systems observed with Spitzer and the Redshift $(z)$ and Early Universe Spectrometer (ZEUS) on the Caltech Submillimeter Observatory (CSO; Stacey et al. 2010; Brisbin et al. 2015).

For GOALS, 5MUSES, and the ZEUS/CSO [C II] sample, $6.2 \mu \mathrm{m}$ luminosities were derived using PAHFIT (Smith et al. 2007) or CAFE (Marshall et al. 2007). It has been shown that PAHFIT-derived PAH line luminosities are greater than the those produced via continuum fitting methods by a factor of $\sim 1.6-1.9$ for $L_{6.2 \mu \mathrm{m}}$ and $L_{11.3 \mu \mathrm{m}}$ (e.g., Sajina et al. 2007; Smith et al. 2007; Pope et al. 2008). This is because PAHFIT is able 
Table 2

Existing Spitzer and Herschel Photometry

\begin{tabular}{|c|c|c|c|c|c|c|c|c|c|c|c|c|}
\hline Target & $\begin{array}{c}3.6 \mu \mathrm{m} \\
(\mu \mathrm{Jy})\end{array}$ & $\begin{array}{c}4.5 \mu \mathrm{m} \\
(\mu \mathrm{Jy})\end{array}$ & $\begin{array}{c}5.8 \mu \mathrm{m} \\
(\mu \mathrm{Jy})\end{array}$ & $\begin{array}{l}8 \mu \mathrm{m} \\
(\mu \mathrm{Jy})\end{array}$ & $\begin{array}{c}16 \mu \mathrm{m} \\
(\mu \mathrm{Jy})\end{array}$ & $\begin{array}{c}24 \mu \mathrm{m} \\
(\mu \mathrm{Jy})\end{array}$ & $\begin{array}{c}70 \mu \mathrm{m} \\
(\mu \mathrm{Jy})\end{array}$ & $\begin{array}{c}100 \mu \mathrm{m} \\
(\mathrm{mJy})\end{array}$ & $\begin{array}{c}160 \mu \mathrm{m} \\
(\mathrm{mJy})\end{array}$ & $\begin{array}{c}250 \mu \mathrm{m}^{\mathrm{a}} \\
(\mathrm{mJy})\end{array}$ & $\begin{array}{c}350 \mu \mathrm{m}^{\mathrm{a}} \\
(\mathrm{mJy})\end{array}$ & $\begin{array}{c}500 \mu \mathrm{m}^{\mathrm{a}} \\
(\mathrm{mJy})\end{array}$ \\
\hline GS IRS20 & $16.91 \pm 0.05$ & $19.89 \pm 0.07$ & $21.59 \pm 0.32$ & $13.42 \pm 0.35$ & $\ldots$ & $275.0 \pm 6.0$ & $3170.0 \pm 590.5$ & $8.32 \pm 0.56$ & $20.15 \pm 1.6$ & $24.78 \pm 2.3$ & $23.82 \pm 3.08$ & $12.49 \pm 5.08$ \\
\hline GS IRS46 & $68.87 \pm 0.06$ & $51.16 \pm 0.08$ & $42.72 \pm 0.47$ & $25.45 \pm 0.45$ & $\ldots$ & $378.0 \pm 6.6$ & $\ldots$ & & $\ldots$ & $13.05 \pm 2.8$ & $15.11 \pm 4.7$ & $13.0 \pm 2.0$ \\
\hline GS IRS50 & $14.9 \pm 0.03$ & $16.7 \pm 0.04$ & $15.88 \pm 0.22$ & $10.49 \pm 0.23$ & $55.6 \pm 6.6$ & $227.0 \pm 8.1$ & $\ldots$ & $1.12 \pm 0.13$ & $3.62 \pm 1.05$ & $5.83 \pm 2.84$ & $6.06 \pm 2.44$ & $4.03 \pm 3.44$ \\
\hline GS IRS52 & $9.93 \pm 0.05$ & $13.47 \pm 0.06$ & $13.15 \pm 0.33$ & $10.7 \pm 0.33$ & $60.9 \pm 8.1$ & $240.0 \pm 8.6$ & $\ldots$ & $\ldots$ & $\ldots$ & $10.06 \pm 3.26$ & $9.05 \pm 4.4$ & $6.86 \pm 3.66$ \\
\hline GS IRS58 & $18.45 \pm 0.04$ & $21.48 \pm 0.06$ & $18.92 \pm 0.27$ & $12.82 \pm 0.31$ & $\ldots$ & $243.0 \pm 8.7$ & $\ldots$ & $1.27 \pm 0.14$ & $3.51 \pm 0.99$ & $8.85 \pm 2.28$ & $11.15 \pm 2.14$ & $5.73 \pm 2.88$ \\
\hline GS IRS61 & $16.21 \pm 0.04$ & $18.73 \pm 0.05$ & $17.05 \pm 0.25$ & $13.37 \pm 0.27$ & $59.6 \pm 5.0$ & $245.0 \pm 8.7$ & $\ldots$ & $3.21 \pm 0.16$ & $5.56 \pm 1.08$ & $5.76 \pm 2.2$ & $3.44 \pm 2.58$ & $4.96 \pm 2.8$ \\
\hline
\end{tabular}

Note.

${ }^{\text {a }}$ Confusion noise for $250 \mu, 350$ and $500 \mu \mathrm{m}$ is $\sim 5 \mathrm{mJy}$ (see Nguyen et al. 2010 for exact values) and has been included in all SED fits. 

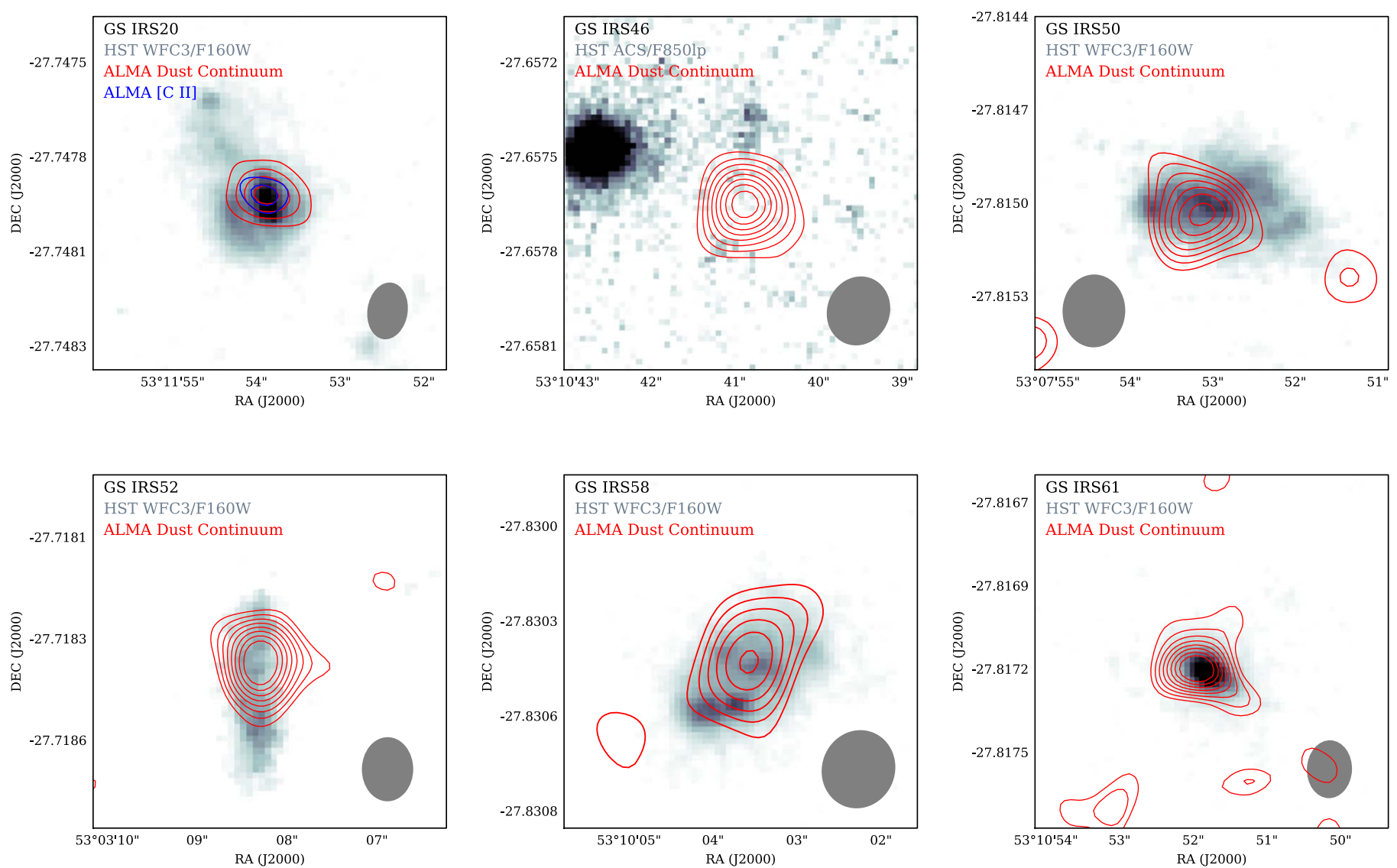

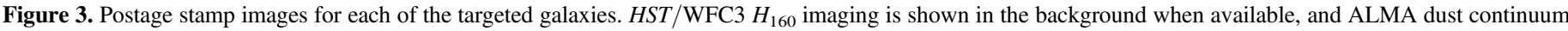

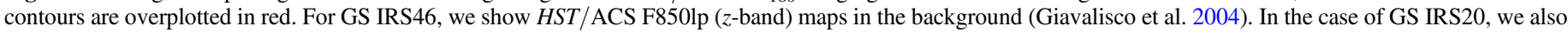
show integrated [C II] emission contours in blue. The ALMA beam is represented by a gray ellipse in all images.

to measure line emission in extended Lorentzian wings whereas continuum fitting methods do not. The $z \sim 2$ Spitzer IRS spectra do not have sufficient $\mathrm{S} / \mathrm{Ns}$ to use PAHFIT reliably, so we instead measure PAH lines using a continuum fitting technique described in Appendix A. In summary, we fit a continuum + line model to isolated 6.2 and $11.3 \mu \mathrm{m}$ regimes allowing the line strength, and galaxy redshift to vary. We also re-measure PAH luminosities in GOALS star-forming galaxies using our method, and divide PAHFIT values by a statistical conversion factor of 1.6 and 2.3 for $L_{6.2 \mu \mathrm{m}}$ and $L_{11.3 \mu \mathrm{m}}$, respectively, to match our quantities derived at higher redshift.

The GOALS sample is nearby and resolved by the Spitzer IRS slit, which is centered on the nuclear region of each galaxy and will not capture the total mid-IR continuum and PAH flux (Armus et al. 2009; Stierwalt et al. 2013). For fair comparison with high- $z$ galaxies that are completely covered by the IRS slit, we correct the PAH line fluxes of GOALS using slitcorrections in Stierwalt et al. (2014) determined from the ratio of total Spitzer IRAC $8 \mu \mathrm{m}$ flux to total IRS $8 \mu \mathrm{m}$ flux. These corrections have a median value of 1.14 and a negligible impact on the average value of GOALS galaxies in the diagnostic plots.

\subsection{SED Fits to Near-IR through Sub-mm Photometry}

Near-IR through sub-mm photometry are shown in Figure 5. For comparison, we overplot the average SED of $z \sim 2$ $\log L_{\mathrm{IR}} / L_{\odot}=12.5$ star-forming galaxies from Kirkpatrick et al. (2015), scaled to best match the observations. The excellent agreement at $5-15 \mu \mathrm{m}$ is due to the fact that our galaxies are part of the sample used in generating the Kirkpatrick et al. (2015) templates, which were normalized in the mid-IR.

To calculate total $8-1000 \mu \mathrm{m}$ IR luminosities, we fit a twotemperature modified blackbody + power-law model between the IRS spectra at rest wavelengths above $9 \mu \mathrm{m}$ out to the far-IR photometry, motivated by Kirkpatrick et al. (2015) who find that a two-temperature model yields good fits to the far-IR SEDs of $z=0.3-2.8$ (U)LIRGs. For all fits, we keep the dust emissivity $\beta$ fixed to a value of 1.5 , and the temperature of the cold dust component fixed at $T_{\text {cold }}=26.1 \mathrm{~K}$ corresponding to the average value of galaxies in the Kirkpatrick et al. (2015) sample with $f_{\mathrm{AGN}, \mathrm{MIR}} \leqslant 0.3$. From the fits, we measure $L_{\mathrm{IR}}$ and the fraction of IR emission originating from the cold dust component $\left(L_{\text {cold }} / L_{\mathrm{IR}}\right)$. Table 4 reports best-fit values for $T_{\text {warm }}$, the modified-blackbody temperature of the warm-dust component, and $L_{\text {cold }}$ with their associated $1 \sigma$ uncertainties for GS IRS20 and GS IRS61. GS IRS46 and GS IRS52 do not have restframe photometry between 30 and $70 \mu \mathrm{m}$, so we determine a best-fit template from the Kirkpatrick et al. (2015) library by matching to the available observations above restframe $9 \mu \mathrm{m}$. The scale-factors for each template are 11.7 and 2.6 in GS IRS46 and GS IRS52, respectively. We then integrate the scaled Kirkpatrick et al. (2015) template to calculate $L_{\mathrm{IR}}$ in these two galaxies.

Models fits are shown in Figure 5 as dashed black lines. Kirkpatrick et al. (2015) find that $L_{\text {cold }} / L_{\mathrm{IR}} \approx 0.5$ on average for $z=0.3-2.8$ (U)LIRGs with $0<f_{\mathrm{AGN}, \mathrm{MIR}}<0.6$. In GS IRS20, the one galaxy where [C II] was detected at high 
Table 3

ALMA Cycle 5 Band 9 Observations: Continuum Imaging and Spectral Line Data

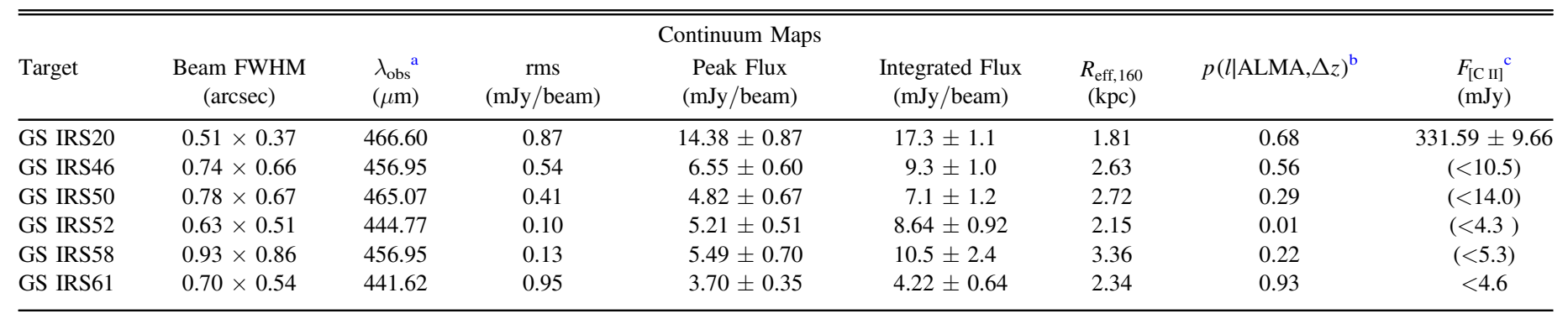

Notes.

a Effective wavelength of collapsed ALMA Band 9 cube.

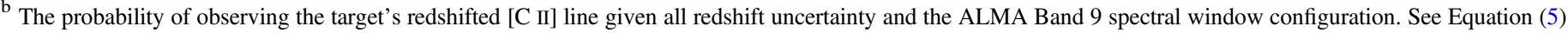
in Section 3.3.

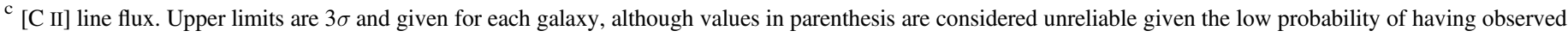
the line.

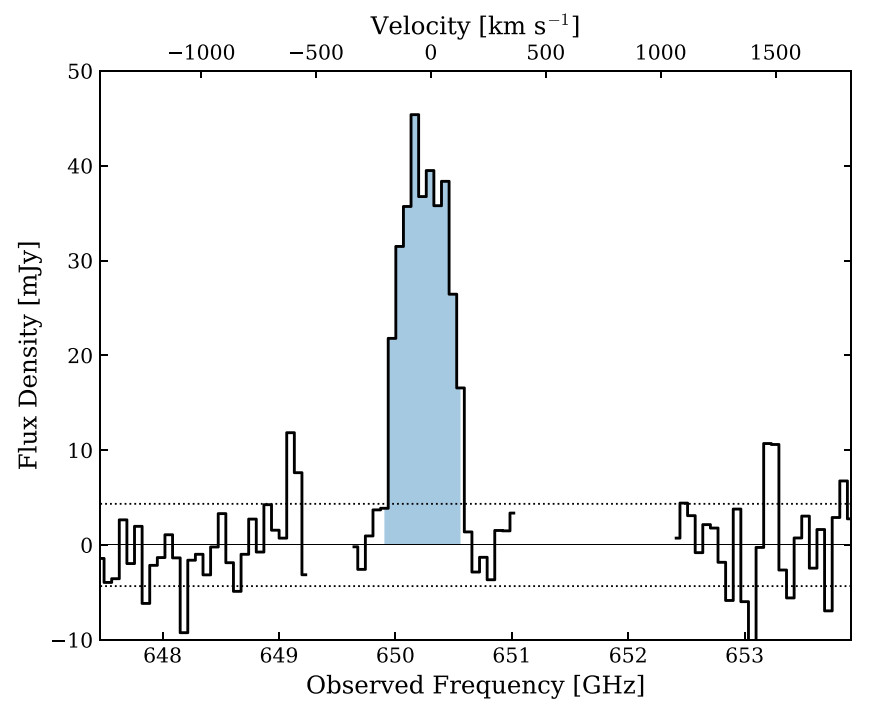

Figure 4. GS IRS20's ALMA Band 9 continuum-subtracted spectrum showing the robust detection of [C II] at $z=1.924$, binned to $30 \mathrm{~km} \mathrm{~s}^{-1}$ resolution. The top axis shows relative velocities in $\mathrm{km} \mathrm{s}^{-1}$ from the line's centroid. The horizontal dotted lines correspond to $\pm 1 \sigma$ noise, and the shaded blue region indicates where line emission was integrated: the peak is detected at an $\mathrm{S} / \mathrm{N}$ of $19.1 \sigma$ and the integrated emission at an $\mathrm{S} / \mathrm{N}$ of $34.3 \sigma$. Gaps in the spectra are due to the observation's spectral window configuration.

significance, and GS IRS61, the target with a secure [C II] upper limit, we measure $L_{\text {cold }} / L_{\mathrm{IR}}=0.28$ and 0.07 , respectively, at the extreme lower end of the distribution for galaxies of similar $f_{\mathrm{AGN}, \mathrm{MIR}}$ and $L_{\mathrm{IR}}$ (Figure 6). GS IRS20 and GS IRS61 deviate from the mean of the Kirkpatrick et al. (2015) sample by $\approx 2.5 \sigma$ and $\approx 12 \sigma$, respectively. Both systems have $T_{\text {warm }}$ comparable to stacked templates of similar $f_{\mathrm{AGN}, \mathrm{MIR}}$ (Table 4 ), indicating that low $L_{\text {cold }} / L_{\mathrm{IR}}$ is driven by an increase in the warm-dust content of these two galaxies, and not a rise in the warm-dust temperature.

\section{Results}

\section{1. [C II] Line Luminosities}

Of the six galaxies targeted with ALMA, we only detect the $158 \mu \mathrm{m}[\mathrm{C}$ II] fine-structure line in one galaxy, GS IRS20, the most IR-luminous source in our sample. For one other galaxy in our sample, GS IRS61, the [C II] line was reliably covered by our ALMA observations $(p(l \mid$ ALMA, $\Delta z)=93 \%)$. For the other targets, our ALMA observations have a probability $>40 \%$ that we missed the redshifted [C II] line given prior redshift uncertainties and the sparse frequency coverage of ALMA spectral windows in Band 9. For the remainder of the paper, we only include GS IRS20 and GS IRS61 in any analysis that involves [C II].

Figure 7 shows the [C II] deficit for low-redshift (U)LIRGs and star-forming galaxies at $z \sim 2-3$ from this sample and the literature. We note that the number of IR-luminous galaxies at $z \gtrsim 4$ with [C II] detections is growing ${ }^{8}$; however, we restrict our current analysis of the high- $z$ landscape to $z \sim 2-3$ to focus on galaxy properties near the cosmic star formation rate density peak. The ratio of $L_{[\mathrm{C} \text { II] }}$ to $L_{\mathrm{IR}}$ in GS IRS20 is comparable to other $z \sim 3$ ALMA [C II] detections from Rybak et al. (2019) and possibly consistent with the extrapolation of the low- $z$ [C II]-deficit to $\log L_{\mathrm{IR}} / L_{\odot} \geqslant 12.5$. GS IRS61 is $\sim 2$ dex below GOALS star-forming galaxies of $\log L_{\mathrm{IR}} / L_{\odot} \approx 12$.

There is a significant offset on the order of 0.5-1.5 dex between $L_{[\mathrm{C} \text { II] }} / L_{\mathrm{IR}}$ at $z=2-3$ found with ALMA and those reported by Stacey et al. (2010) and Brisbin et al. (2015) using ZEUS/CSO (blue in Figure 7). The spectral resolution of ZEUS is $150-300 \mathrm{~km} \mathrm{~s}^{-1}$, comparable to the expected line width of $[\mathrm{CII}]$ emission in some cases, making the flux measurements sensitive to the number of spectral pixels included when integrating a low-S/N line. We re-calculate all ZEUS/CSO [C II] luminosities using only the peak pixel flux assuming a line width of $150-300 \mathrm{~km} \mathrm{~s}^{-1}$. After these corrections, the 0.5-1.5 dex offset between ALMA and ZEUS observations in $z \sim 2$ SFGs persists. There are multiple factors that could contribute to this offset, including physical variations in $[\mathrm{C} \mathrm{II}] / L_{\mathrm{IR}}$ with star-formation-rate surface density (e.g., Díaz-Santos et al. 2017; Smith et al. 2017), or observational limitations such as large beam sizes, lower spectral resolution,

\footnotetext{
8 Exempli gratia, Gallerani et al. (2012), Walter et al. (2012), Riechers et al (2013), Bussmann et al. (2013), Rawle et al. (2014), De Breuck et al. (2014), Maiolino et al. (2015), Capak et al. (2015), Oteo et al. (2016), Pentericci et al. (2016), Carniani et al. (2017), Jones et al. (2017), Matthee et al. (2017), Smit et al. (2018), Carniani et al. (2018), Gullberg et al. (2018), Decarli et al. (2018), Hashimoto et al. (2018), Le Fèvre et al. (2019), Tadaki et al. (2019), Hashimoto et al. (2019).
} 

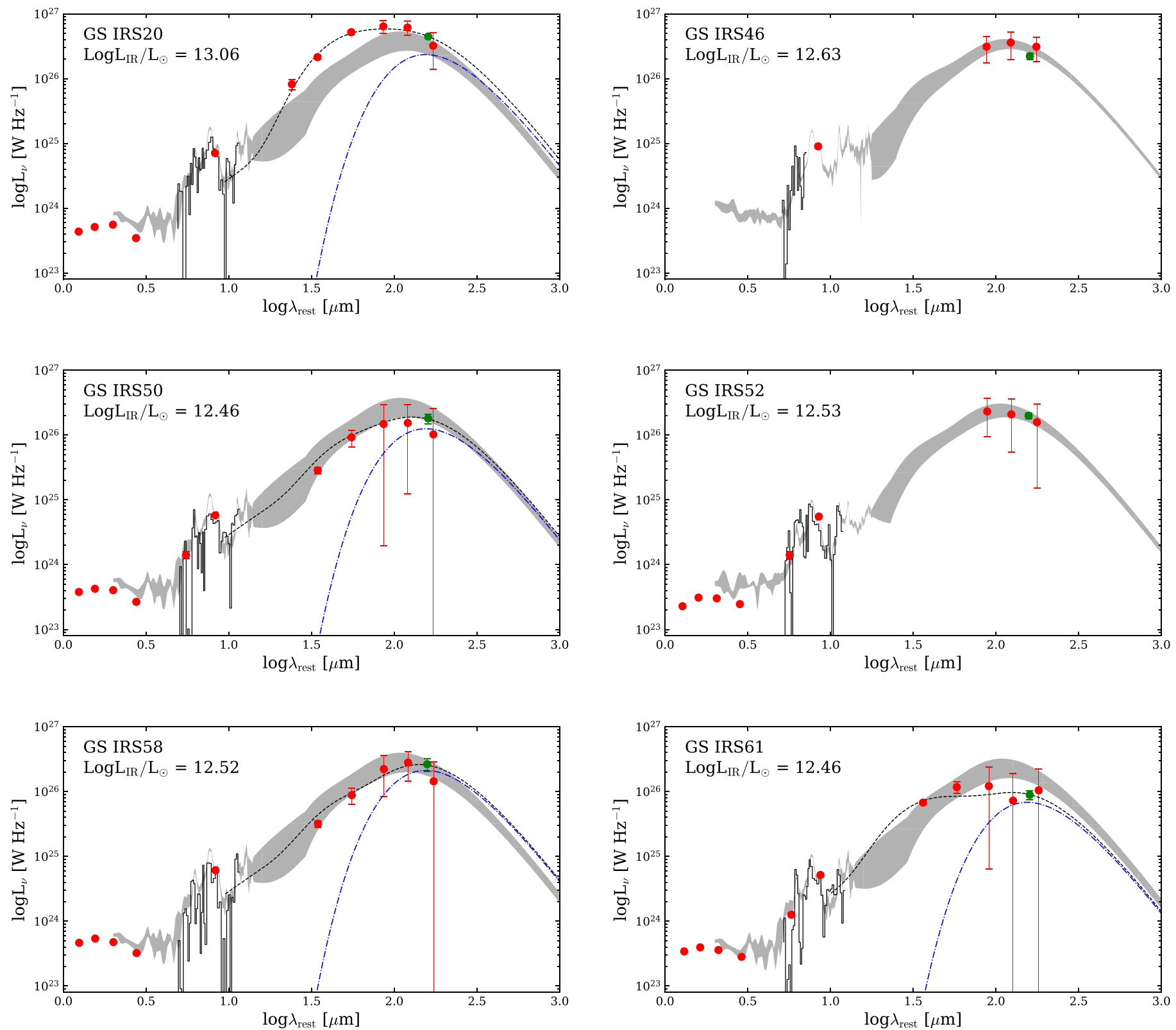

Figure 5. Multiwavelength photometry and spectra for each ALMA target. Shaded gray SEDs correspond to the best-fit $z \sim 2$ template SED from the empirical library presented in Kirkpatrick et al. (2015). The solid black line corresponds to a two-temperature modified blackbody + near-IR power-law fit, which we integrate to estimate $L_{\mathrm{IR}}$ for each target. The dotted-dashed blue line is the cold dust component from this fit that we integrate to calculate $L_{\text {cold. }}$. Photometry in red correspond to Spitzer and Hershel observations. ALMA dust continuum is shown in green, and Spitzer IRS spectra are overplotted in black. We do not fit the blackbody + powerlaw models to GS IRS46 and GS IRS52 because these galaxies lack observations between restframe 30-70 $\mu \mathrm{m}$.

and flux calibration uncertainties on the order of $30 \%$ (Brisbin et al. 2015).

\subsection{PAH Properties}

The relationship between PAH emission and dust emission evolves with redshift and is likely related to a number of factors, including $f_{\mathrm{AGN}, \mathrm{MIR}}$, SFR, and the number of PDRs per unit molecular gas mass (Smith et al. 2007; Pope et al. 2013). Star-forming galaxies in our sample at $z \sim 2$ have $6.2 \mu \mathrm{m} \mathrm{PAH}$ luminosities 0.3 dex brighter than local (U)LIRGs of comparable $L_{\mathrm{IR}}$ after accounting for the differences in $L_{6.2 \mu \mathrm{m}}$ measurement techniques (see Section 3.4) but follow a deficit in PAH emission toward higher $L_{\mathrm{IR}}$ whose magnitude of decline is equal to or greater than the deficit between other farIR fine-structure lines and $L_{\mathrm{IR}}$ (Pope et al. 2008; Sajina et al. 2008; Graciá-Carpio et al. 2011; Pope et al. 2013; Stierwalt et al. 2014; Shipley et al. 2016; Cortzen et al. 2019). In addition to being a function of $L_{\mathrm{IR}}, L_{6.2 \mu \mathrm{m}} / L_{\mathrm{IR}}$ also changes with $z$ (e.g., Pope et al. 2013), as demonstrated in Figure 8 (left panel), which shows the ratio of $L_{6.2 \mu \mathrm{m}}$ to $L_{\mathrm{IR}}$ for low- and high- $z$ starforming galaxies. Galaxies at $z \sim 2$ in our sample are brighter in $L_{\mathrm{IR}}$ by a factor of $\gtrsim 0.5$ dex compared to low- $z$ (U)LIRGs of comparable $L_{6.2 \mu \mathrm{m}} / L_{\mathrm{IR}}$; changes in either/both of $L_{6.2 \mu \mathrm{m}}$ and 


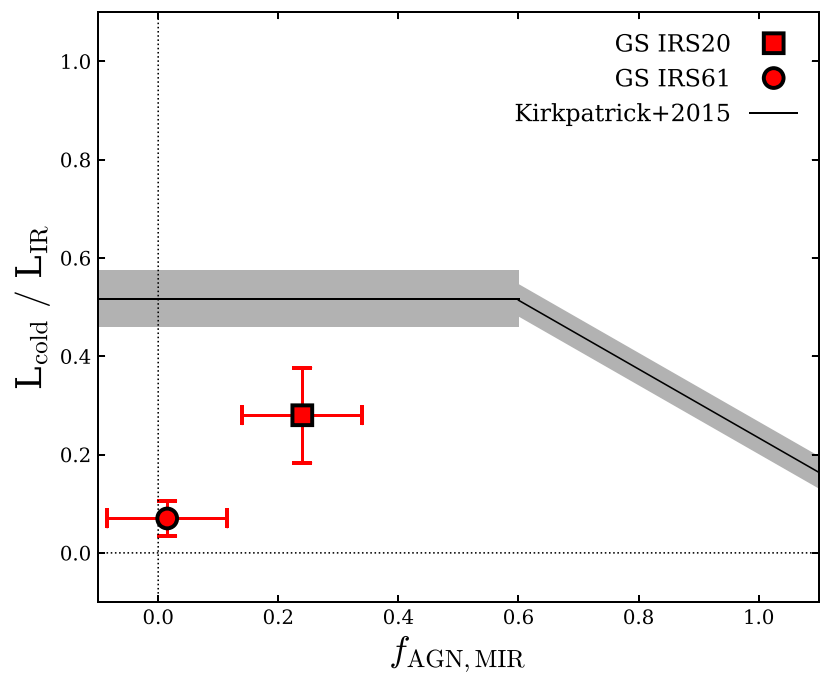

Figure 6. The ratio of cold dust emission, $L_{\text {cold }}$, to $L_{\mathrm{IR}}$ as a function of $f_{\mathrm{AGN}, \mathrm{MIR}}$, the AGN contribution to emission at mid-IR wavelengths. $L_{\text {cold }}$ comes from integrating the cold dust component in our two-temperature SED fits shown in Figure 5. Our data is shown in red. The solid black line indicates the best-fit trend for 343 (U)LIRGs between $z=0.3-2.8$ from Kirkpatrick et al. (2015), with the $1 \sigma$ uncertainty shaded in gray.

Table 4

Derived Parameters from IR-SED Fits

\begin{tabular}{lccc}
\hline \hline Target & $T_{\text {cold }}(\mathrm{K})$ & $T_{\text {warm }}(\mathrm{K})$ & $L_{\text {cold }} / L_{\mathrm{IR}}$ \\
\hline GS IRS20 & 26.1 (fixed) $^{\mathrm{a}}$ & $57 \pm 1$ & $0.28 \pm 0.10$ \\
GS IRS61 & 26.1 (fixed) $^{\mathrm{a}}$ & $59 \pm 2$ & $0.07 \pm 0.04$ \\
\hline MIR0.0 $^{\mathrm{b}}$ & $25.7 \pm 0.6$ & $66 \pm 2$ & $0.51 \pm 0.04$ \\
MIR0.2 $^{\mathrm{b}}$ & $24.6 \pm 1.3$ & $62 \pm 1$ & $0.44 \pm 0.06$ \\
\hline
\end{tabular}

Notes.

${ }^{\mathrm{a}} T_{\text {cold }}$ was fixed in the SED fits of both GS IRS20 and GS IRS61 to the average of galaxies in the Kirkpatrick et al. (2015) sample with $f_{\text {AGN,MIR }} \leqslant 0.3$.

${ }^{\mathrm{b}}$ Empirical templates from Kirkpatrick et al. (2015) of comparable $f_{\mathrm{AGN}, \mathrm{MIR}}$ to GS IRS20 and GS IRS61. MIR0.0 and MIR0.2 correspond to $f_{\mathrm{AGN}, \mathrm{MIR}}=0.0$ and $f_{\mathrm{AGN}, \mathrm{MIR}}=0.2$, respectively.

$L_{\mathrm{IR}}$ could drive the difference between low-redshift and $z \sim 2$ galaxies in Figure 8. In any case, this trend persists if we instead use the ratio of $11.3 \mu \mathrm{m}$ PAH luminosity to $L_{\mathrm{IR}}$ as well as values for $L_{6.2 \mu \mathrm{m}}$ in GOALS measured using our method described in Appendix A.

Díaz-Santos et al. (2017) show that the IR surface density is a good predictor of physical PDR conditions such as gas density and incident radiation field strength. Furthermore, spatially resolved studies of nearby and $z \sim 3$ star-forming galaxies have shown the star formation rate surface density $\left(\Sigma_{\mathrm{SFR}}\right)$ to be a major driver of the [C II]-deficit (Díaz-Santos et al. 2014; Smith et al. 2017; Rybak et al. 2019). In light of these results, and without spatial information at shorter wavelengths more aptly suited for tracing $\Sigma_{\mathrm{SFR}}$, we calculate $\Sigma_{\mathrm{IR}}=\left(L_{\mathrm{IR}} / 2\right) / \pi R_{\mathrm{eff}, 160}^{2}$, the effective IR surface density using $R_{\text {eff, } 160}$ as measured with ALMA for our sample and Herschel
PACS in GOALS. Figure 8 (right panel) demonstrates that the offset between high- and low- $z$ galaxies in $L_{6.2 \mu \mathrm{m}} / L_{\mathrm{IR}}$ disappears when plotted against $\Sigma_{\mathrm{IR}}$.

\subsection{The Ratio of [C II] to PAH Luminosity}

Figure 9 shows $L_{\left[\mathrm{C}_{\mathrm{II}}\right]} / L_{6.2 \mu \mathrm{m}}$ as a function of $\Sigma_{\mathrm{IR}}$ for our sample, and local (U)LIRGs. GOALS star-forming galaxies $\left(\mathrm{EW}_{6.2 \mu \mathrm{m}} \geqslant 0.5 \mu \mathrm{m}\right)$ have a tight ratio of $L_{[\mathrm{C} \mathrm{II}} / L_{6.2} \mu \mathrm{m}$ with a dispersion of $0.18 \mathrm{dex}$, less than the $\sim 0.3$ dex dispersion observed in both $L_{[\mathrm{CI}]} / L_{\mathrm{IR}}$ and $L_{6.2 \mu \mathrm{m}} / L_{\mathrm{IR}}$. We fit a linear relation to star-forming GOALS galaxies on Figure 9 and find that $L_{[\mathrm{C} \text { II }} / L_{6.2 \mu \mathrm{m}}$ and $\Sigma_{\mathrm{IR}}$ anticorrelate with a slope of $-0.23 \pm 0.08$ and zero-point $2.2 \pm 0.9$. Although high-redshift observations remain limited by small sample statistics, the spread in $L_{[\mathrm{C} \text { II] }} / L_{6.2 \mu \mathrm{m}}$ between GS IRS20 and GS IRS61 at $z \sim 2$ is 0.98 dex, five times greater than the dispersion of local star-forming (U)LIRGs, an observation that holds regardless of how the PAH luminosities are measured in GOALS (see Section 3.4).

GS IRS20 and GS IRS61 are $\sim 0.67$ and $\geqslant 1.65$ dex, respectively, below the mean of $L_{[\mathrm{CI}} / L_{6.2 \mu \mathrm{m}}$ observed in GOALS star-forming galaxies, after accounting for the differences in how the PAHs were measured. GS IRS20 is possibly consistent with the extrapolation of the low- $z$ negative trend between $L_{[\mathrm{C} \text { II] }} / L_{6.2 \mu \mathrm{m}}$ and $\Sigma_{\mathrm{IR}}$ beyond the most compact GOALS star-forming galaxy; however, this cannot explain the extremely low ratio observed in GS IRS61. GS IRS61 shows no indication of a deeply buried AGN (Figure 2), and there is a low probability that we missed the redshifted [C II] line (see Appendix B). The dust continuum is marginally more extended than the ALMA beam and would have to be extraordinarily compact $\left(\Sigma_{\mathrm{IR}}\right.$ would have to increase by $>2$ orders of magnitude) to be consistent with the extrapolated low- $z$ trend. For these reasons, GS IRS61 is likely a highly unusual source when compared to low- $z$ star-forming galaxies of comparable $\Sigma_{\mathrm{IR}}$.

If we assume that the relevant physical parameters of the $z \sim 2$ galaxies are drawn from the same distribution that is observed in GOALS, then GS IRS20 and GS IRS61 would be $\sim 3 \sigma$ and $\gtrsim 6 \sigma$ below the low $z$ mean. The likelihood of observing two galaxies at $3 \sigma$ and $6 \sigma$ from the norm is $\approx 10^{-11}$. Therefore, the offset in $L_{[\mathrm{C} \text { II] }} / L_{6.2 \mu \mathrm{m}}$ between low- $z$ (U)LIRGs and what we measure in our sample may relate to changes in the physical ISM conditions.

\section{Discussion}

\subsection{PAH Heating versus Far-IR Cooling}

We find a difference in the ratio of [C II] to PAH emission between local ULIRGs and observations of two $z \sim 2$ dusty star-forming galaxies including one upper limit (Figure 9), which could be due to changes in heating and cooling mechanisms. As opposed to being scaled up versions of nearby star-forming galaxies, starbursts at earlier times may exhibit evolution in their ISM conditions. While the behavior of both PAH and [C II] changes at a low metallicity (Croxall et al. 2017; Shivaei et al. 2017), we do not expect this to affect our massive $\left(\log M_{*} / M_{\odot} \sim 11\right) z \sim 2$ galaxies given the $z \sim 2$ mass-metallicity relation (Sanders et al. 2015). 
PAHs and other small grains are important sources of photoelectrons in PDRs (e.g., Bakes \& Tielens 1994), and the ratio of far-IR line to PAH emission is sensitive to the photoelectric heating efficiency $\left(\epsilon_{\mathrm{PE}}\right)$ of the PDR gas. As noted by Helou et al. (2001), $L_{\mathrm{PAH}}$ (or $L_{6.2 \mu \mathrm{m}}$ ) may be more appropriate normalization factors for $L_{[\mathrm{C} \text { II] }}$ than $L_{\mathrm{IR}}$ given the direct relationship with $\epsilon_{\mathrm{PE}}$ :

$$
\frac{L_{[\mathrm{C} \text { II }]}+L_{[\mathrm{O} \mathrm{I}]}}{L_{\mathrm{PAH}}}=\left(\eta_{[\mathrm{C} \mathrm{II}]}+\eta_{[\mathrm{O} \mathrm{I}]} \epsilon_{\mathrm{PE}} \approx \frac{\Gamma_{\mathrm{gas}}^{e^{-}}}{\Gamma_{\mathrm{dust}}^{\mathrm{PAHs}}}\right.
$$

where following Croxall et al. (2012), $\eta_{[\mathrm{C} \mathrm{II}]}$ and $\eta_{[\mathrm{O} \mathrm{I}]}$ represent the relative contribution of the two principal cooling channels to the total gas cooling. $\Gamma_{\text {gas }}^{e^{-}}$is the total gas heating via photoelectrons, and $\Gamma_{\text {dust }}^{\mathrm{PAHs}}$ the total dust heating accounted for by PAHs. Cooling from other far-IR such as [C I] and [Si II] are assumed to be negligible (i.e., $\eta_{[\mathrm{C} \mathrm{II]}}+\eta_{[\mathrm{O} \text { I] }} \sim 1$ ).

Assuming that the $6.2 \mu \mathrm{m}$ PAH feature linearly scales with total PAH luminosity (e.g., Smith et al. 2007), and the fraction of [C II] emission originating from PDRs is roughly constant, then the ratio $L_{[\mathrm{C} \mathrm{II}} / L_{6.2 \mu \mathrm{m}}$ probes the difference of photoelectric efficiency and normalized cooling via [OI]. Knowing that the ratio of [O I] emission to [C II] emission of PDR origin varies by an order of magnitude in nearby (U)LIRGs (Díaz-Santos et al. 2017), the location of GS IRS20 and GS IRS61 on Figure 9 could be interpreted as evidence for enhanced [O I] cooling in these galaxies if the total $\epsilon_{\mathrm{PE}}$ is constant. Díaz-Santos et al. (2017) demonstrate that [O I]/[C II] correlates with gas and dust temperature within PDRs, and $[\mathrm{O} \mathrm{I}] /[\mathrm{CII}]>1$ where dust temperatures exceed $\sim 35 \mathrm{~K}$. Indeed, warm-dust blackbodies ( $T_{\text {warm }} \sim 60 \mathrm{~K}$ ) dominate the IR SEDs of both GS IRS20 and GS IRS61 $\left(L_{\text {cold }} / L_{\mathrm{IR}} \lesssim 0.3\right.$, Table 4$)$, consistent with enhanced PDR cooling through [O I] emission. Moreover, our $z \sim 2$ sample has high $\Sigma_{\text {IR }}$ compared to the average of GOALS (Figure 8 Right), implying more star formation in smaller volumes. In such physical conditions, PDR densities are expected to be higher and exposed to more intense radiation fields where [O I] naturally arises as the dominant cooling channel (Díaz-Santos et al. 2017). If the positions of GS IRS20 and GS IRS61 on Figure 9 are solely due to enhanced [O I] cooling $\left(\epsilon_{\mathrm{PE}}=\right.$ constant $)$, then we calculate $L_{[\mathrm{O} \text { I] }}=7 \times 10^{9} L_{\odot}$ and $L_{[\mathrm{O}]} \gtrsim 10^{10} L_{\odot}$ for these two galaxies, respectively, in order to bring both in line with the GOALS sample. In this scenario, $L_{[\mathrm{O} \text { I] }} / L_{[\mathrm{C} \mathrm{II]}} \sim 5$ in GS IRS20 and [O I] dominates farIR line cooling in both galaxies.

Alternatively, low $L_{[\mathrm{C} \mathrm{II}]} / L_{6.2 \mu \mathrm{m}}$ could indicate a low $\epsilon_{\mathrm{PE}}$ by Equation (2) if [O I] emission is not significantly enhanced in GS IRS20 and/or GS IRS61. We speculate that a decrease in the photoelectric efficiency in high- $z$ dusty star-forming galaxies could play a role in enhancing star formation rates compared to the galaxy main sequence by reducing the coupling efficiency between interstellar radiation fields and gas heating. In other words, the colder ISM phases become less susceptible to temperature increases via stellar feedback as the reservoir of electrons in PAHs is diminished. Consequently, galaxies above the main sequence would not exhibit strong farIR line cooling at higher SFRs, as has been observed locally and tentatively at high- $z$ (Díaz-Santos et al. 2017; Zanella et al.

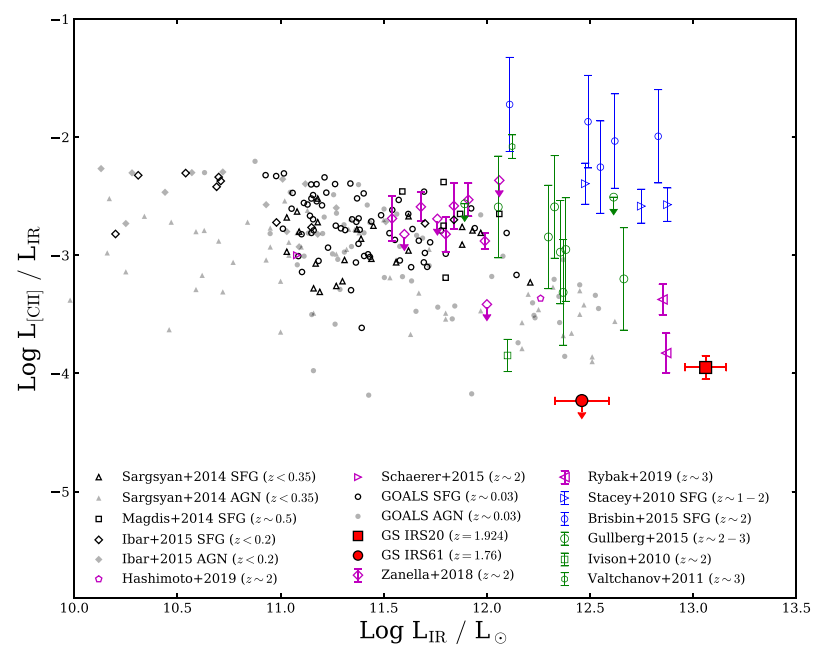

Figure 7. The ratio of [C II] luminosity to $L_{\mathrm{IR}}$ in low- and $z \sim 2-3$ star-forming galaxies and AGNs. We show local star-forming galaxies as black open symbols and low- $z$ AGNs as gray symbols. At $z=2-3$, we show both starforming and AGN systems with colored symbols: ALMA-derived [C II] luminosities are shown in red (this work) and magenta. Blue symbols indicate [C II] observations from ZEUS/CSO, which we re-calculate as described in Section 4.1. Green symbols correspond to galaxies targeted using APEX and Herschel, including the lensed SPT DSFG sample of Gullberg et al. (2015), which we de-magnify using their average magnification factor of 14.1 . We include AGNs in this figure to demonstrate the spread in $L_{[\mathrm{C} \mathrm{II}} / L_{\mathrm{IR}}$ observed in all galaxies; however, we emphasize that this work focuses on the range of star formation properties in galaxies without AGNs.

2018). A comprehensive study of far-IR fine-structure emission lines combined with mid-IR PAH spectra is needed to test this hypothesis, and the nature of gas heating and cooling at $z \sim 2$ will be a function of $\epsilon_{\mathrm{PE}}, \eta_{[\mathrm{C} \mathrm{III}}$, and $\eta_{[\mathrm{O} \mathrm{I}]}$. Systematically low $\epsilon_{\mathrm{PE}}$ in dusty star-forming galaxies at $z \sim 2$ would be associated with $[\mathrm{OI}] /[\mathrm{C} \mathrm{II}] \sim 1$ in a statistical sample controlled for $f_{\mathrm{AGN}, \mathrm{MIR}}$, whereas $[\mathrm{O} \mathrm{I}] /[\mathrm{C} \mathrm{II}]>1$ would favor higher density PDRs with more [O I] cooling. These far-IR cooling line ratios will be key for accessing the physical conditions in which most of the universe's stellar mass was formed.

\subsection{Differences Between GS IRS20 and GS IRS61}

The data in hand portrays an interesting dichotomy of ISM conditions between GS IRS20 and GS IRS61. A 1 dex difference in $L_{[\mathrm{C} \mathrm{II}} / L_{6.2 \mu \mathrm{m}}$ exists between the two galaxies, and is likely a function of PAH ionization state and therefore $\epsilon_{\mathrm{PE}}$. Whereas the $6.2 \mu \mathrm{m}$ feature traces ionized PAHs, the $11.3 \mu \mathrm{m}$ complex arises from neutral PAHs yet to lose their surface electrons (Tielens 2008). As a result, the ratio of $L_{6.2 \mu \mathrm{m}} / L_{11.3 \mu \mathrm{m}}$ is sensitive to the PAH ionization fraction in a galaxy and also to changes in the grain size distribution as observed near the nuclei of AGNs (Smith et al. 2007; Tielens 2008). Figure 10 shows this ratio as a function of $\Sigma_{\mathrm{IR}}$ for GOALS and our sample at $z \sim 2$. GS IRS20 has a PAH line ratio near the local average, as may be expected if star formation in this merging galaxy is proceeding in a comparable manner to what is found in GOALS, which are mostly mergers themselves. On the other hand, GS IRS61 has the highest ratio of $L_{6.2 \mu \mathrm{m}} / L_{11.3 \mu \mathrm{m}}$ among galaxies at low and high redshifts. This is consistent with the location of GS IRS61 in Figure 9: an increase in PAH ionization would lower $\epsilon_{\mathrm{PE}}$, 

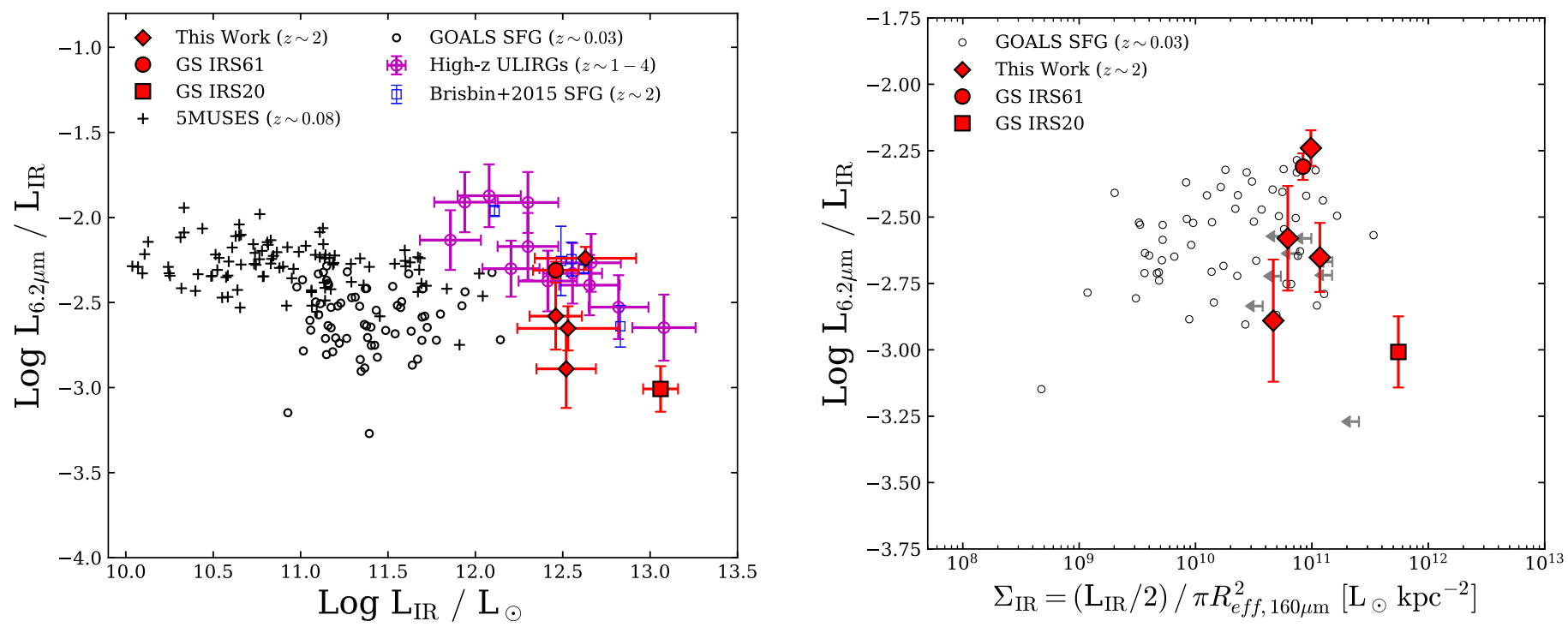

Figure 8. Left panel: the ratio of $L_{62 \mu \mathrm{m}}$ to $L_{\mathrm{IR}}$ in low- and high-redshift IR-luminous galaxies as a function of $L_{\mathrm{IR}}$. Local galaxies are taken from 5MUSES (Wu et al. 2010) and the GOALS sample (Díaz-Santos et al. 2013, 2014, 2017; Stierwalt et al. 2014) and follow the color scheme of previous figures. High-redshift galaxies are represented with colored symbols: red corresponds to our sample and blue indicates galaxies from Brisbin et al. (2015). (U)LIRGs shown in purple are tabulated in Pope et al. (2013). Right panel: the ratio of $L_{6.2 \mu \mathrm{m}}$ to $L_{\mathrm{IR}}$ vs. IR surface density. Upper limits on $\Sigma_{\mathrm{IR}}$ for GOALS galaxies smaller than the Herschel beam are shown in gray. The offset between low- and high-redshift galaxies observed in the left panel is removed when normalizing $L_{\mathrm{IR}}$ by the cold dust surface density traced by restframe $160 \mu \mathrm{m}$ emission with ALMA and Herschel.

decoupling $\mathrm{PAH}$ and $[\mathrm{C} \mathrm{II}]$ emission to produce the extreme deficit in $L_{[\mathrm{C} \mathrm{II]}} / L_{6.2 \mu \mathrm{m}}$ observed.

The only low- $z$ galaxies within $1 \sigma$ of GS IRS61 on Figure 10 are a handful of GOALS AGNs, and the ratio of $L_{6.2 \mu \mathrm{m}} / L_{11.3 \mu \mathrm{m}}$ appears larger than most star-forming GOALS galaxies, even after correcting for PAH extinction (see Figure 2 of Stierwalt et al. 2014). Whether or not this is common at high redshift remains to be explored; however, the scatter in $L_{6.2 \mu \mathrm{m}} / L_{11.3 \mu \mathrm{m}}$ we measure at $z \sim 2$ is nearly three times larger than what is seen in the GOALS star-forming sample. Although, we note that error bars at higher $z$ are large. While both GS IRS20 and GS IRS61 have comparable far-IR colors, GS IRS61 has a lower $L_{\text {cold }} / L_{\text {IR }}$ (Figure 6), indicating warmer dust conditions dominating the galaxy, consistent with low $\epsilon_{\mathrm{PE}}$ as larger dust grains absorb more of the incident radiation field in PDRs. The parameter space of PAH line ratios at cosmic noon has yet to be statistically explored and may prove key for our understanding of dust properties and the link between stellar radiation fields and the ISM at the peak epoch of galaxy evolution.

\subsection{Future Outlook}

Testing the nature of gas heating and cooling in the ISM of high-redshift galaxies will be possible with future ALMA observations targeting [C II] in IRS sources. Mid-IR spectra are crucial for constraining $f_{\mathrm{AGN}, \mathrm{MIR}}$, from which the properties of star formation at high- $z$ can be reliably characterized in the absence of or presence of an AGN. Spitzer's cryogenic lifetime has ended, so the number of galaxies with available mid-IR spectra is currently limited. Future surveys with JWST/MIRI will re-open the mid-IR universe at high spectral sensitivity.

Pending the launch of JWST, ALMA can continue targeting IRS galaxies to explore the relationship between [C II] and $\mathrm{PAH}$ emission as a function of $L_{\mathrm{IR}}$ and $f_{\mathrm{AGN} \text { MIR }}$. Understanding the intrinsic scatter in these relations will be crucial when designing efficient surveys that maximize the science potential of JWST and key for understanding the physics of gas heating and cooling in the early universe, which observations with future facilities like Origins Space Telescope $e^{9}$ or SPICA ${ }^{10}$ will revolutionize.

\section{Summary and Conclusions}

We have observed [C II] emission in a sample of $z \sim 2$ starforming galaxies with existing detections of PAH dust emission in order to explain the balance of heating and cooling in the ISM and how it may be different from $z \sim 0$. Our main conclusions are as follows:

1. We detect the dust continuum near the peak of the IR $\operatorname{SED}\left(\lambda_{\text {rest }} \sim 160 \mu \mathrm{m}\right)$ in all six targets. After correcting for known astrometry offsets between ALMA and HST, the position of the dust continuum emission coincides with the restframe optical light in all but GS IRS46. Our most luminous target GS IRS20 is classified as a merger and is a clear starburst on the main-sequence diagnostic diagram.

2. We detect [C II] in one target, GS IRS 20 at high $\mathrm{S} / \mathrm{N}$ of 34. The bright $[\mathrm{C} \mathrm{II}]$ emission and interesting optical morphology makes this an excellent target for follow-up ALMA observations to study its gas dynamics at higher spatial resolution. We place a deep upper limit on $L_{[\mathrm{C} \text { II] }}$ in one other galaxy, GS IRS61, after calculating the probability the redshifted [C II] line fell into our ALMA bandpass tuning. For other targets in our sample, our observations likely missed the galaxy's [C II] line. Our $z \sim 2$ galaxies follow the [C II]-deficit relation observed for nearby (U)LIRGs, as found by several other $z \sim 2-3$ studies.

\footnotetext{
9 https://origins.ipac.caltech.edu/

${ }^{10}$ https://spica-mission.org/
} 


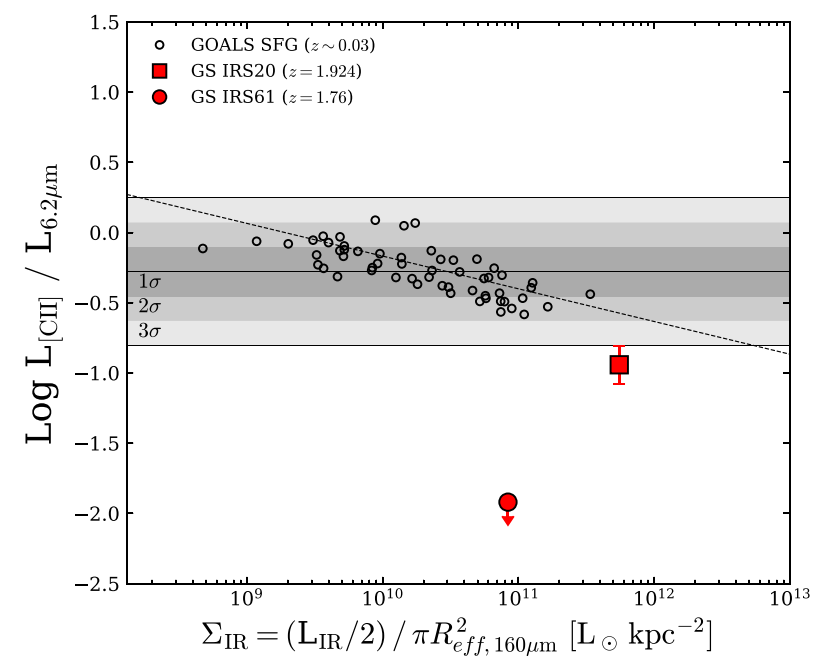

Figure 9. The ratio of [C II] luminosity to $6.2 \mu \mathrm{m}$ PAH luminosity in lowredshift and $z \sim 2$ IR-luminous star-forming galaxies as a function of IR surface density. The effective radius for all sources shown is calculated at a restframe $160 \mu \mathrm{m}$ continuum. The gray shaded regions contain the $1 \sigma, 2 \sigma$, and $3 \sigma$ dispersions around the mean of $L_{[\mathrm{C} \mathrm{I}]} / L_{6.2} \mu \mathrm{m}$ in star-forming GOALS galaxies. The dotted black line corresponds to the best-fit trend in GOALS, and has a slope of $-0.23 \pm 0.08$ and zero-point equal to $2.2 \pm 0.9$. The dearth of high- $z$ points on this Figure demonstrates the need for more observations of [C II], PAH, and IR size measurements in the same galaxies.

3. As found in previous studies, our $z \sim 2$ galaxies and other high- $z$ samples show decreasing $L_{6.2} \mu \mathrm{m} / L_{\mathrm{IR}}$ with $L_{\mathrm{IR}}$. Star-forming galaxies at $z \sim 2$ have more $\mathrm{PAH}$ emission per unit $L_{\mathrm{IR}}$ compared to low- $z$ star-forming galaxies of comparable $L_{\mathrm{IR}}$; however, this offset disappears when comparing $L_{6.2 \mu \mathrm{m}} / L_{\mathrm{IR}}$ in all galaxies as a function of IR surface density.

4. We explore the balance of heating and cooling in the ISM by looking at the ratio of [C II] to PAH luminosity. For nearby (U)LIRGs, this ratio is relatively tight as a function of $L_{\mathrm{IR}}$. Our $z \sim 2$ galaxies are low relative to this relation. This may be because of warmer environments, suppressed photoelectric efficiencies in PDR gas, and/or the importance of cooling from other far-IR lines such as [O I] at $z \sim 2$. GS IRS61, the galaxy with the lowest [C II]/PAH, shows evidence for high PAH ionization, consistent with inefficient gas heating in PDR regions.

We caution that our study shows that [C II] and $\mathrm{PAH}$ emission may not have a simple relation to $L_{\mathrm{IR}}$, and therefore, SFR, in $z \sim 2$ dusty star-forming galaxies. Further observations are needed to validate our results and test the ideas of warmer dust environments and additional cooling channels. These can be obtained by getting more [C II] detections of galaxies with existing PAH measurements from Spitzer/IRS or from future programs tracing the mid-IR and far-IR lines with JWST and ALMA.

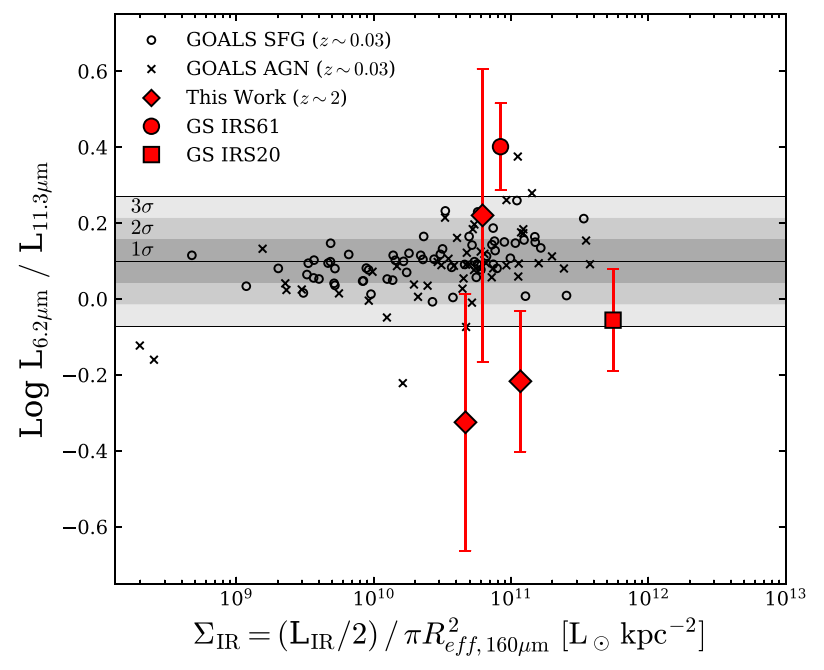

Figure 10. The ratio of $L_{6.2 \mu \mathrm{m}}$ to $L_{11.3 \mu \mathrm{m}}$ vs. effective IR surface density calculated at restframe $160 \mu \mathrm{m}$. The color scheme follows previous figures. $L_{6.2 \mu \mathrm{m}} / L_{11.3 \mu \mathrm{m}}$ does not change drastically with $\Sigma_{\mathrm{IR}}$ in star-forming GOALS galaxies, with minimal $1 \sigma$ scatter $\sim 0.06$ dex shaded in gray about an average value of 0.08 .

We are very grateful to the referee for the detailed comments and suggestions that significantly improved this work. J.M. and A.P. acknowledge D. Brisbin and C. Ferkinhoff for assistance in interpreting ZEUS [C II] results. J.M. acknowledges I. Yoon, J. Braatz, and J. Thorley for their insights into ALMA data reduction. We thank J. D. Smith for his helpful discussion. T. D-S. acknowledges support from the CASSACA and CONICYT fund CAS-CONICYT Call 2018. Support for this work was provided by the NSF through award SOSPA5-008 from the NRAO and through the Massachusetts Space Grant Consortium (MASGS). The National Radio Astronomy Observatory is a facility of the National Science Foundation operated under cooperative agreement by Associated Universities, Inc. This paper makes use of the following ALMA data: ADS/JAO.ALMA\#2017.1.01347.S. ALMA is a partnership of ESO (representing its member states), NSF (USA) and NINS (Japan), together with NRC (Canada), MOST and ASIAA (Taiwan), and KASI (Republic of Korea), in cooperation with the Republic of Chile. The Joint ALMA Observatory is operated by ESO, AUI/NRAO and NAOJ.

\section{Appendix A \\ PAH-Derived Redshifts and Luminosities}

In this section, we describe our method for measuring the PAH redshifts and luminosities that employs MCMC to fully capture the uncertainties. Spitzer IRS mid-IR spectra are shown in Figure 2, which we use to calculate the redshift probability distribution function $p(z)$ and $\mathrm{PAH}$ line luminosities for galaxies in our sample. Restframe mid-IR wavelengths are 

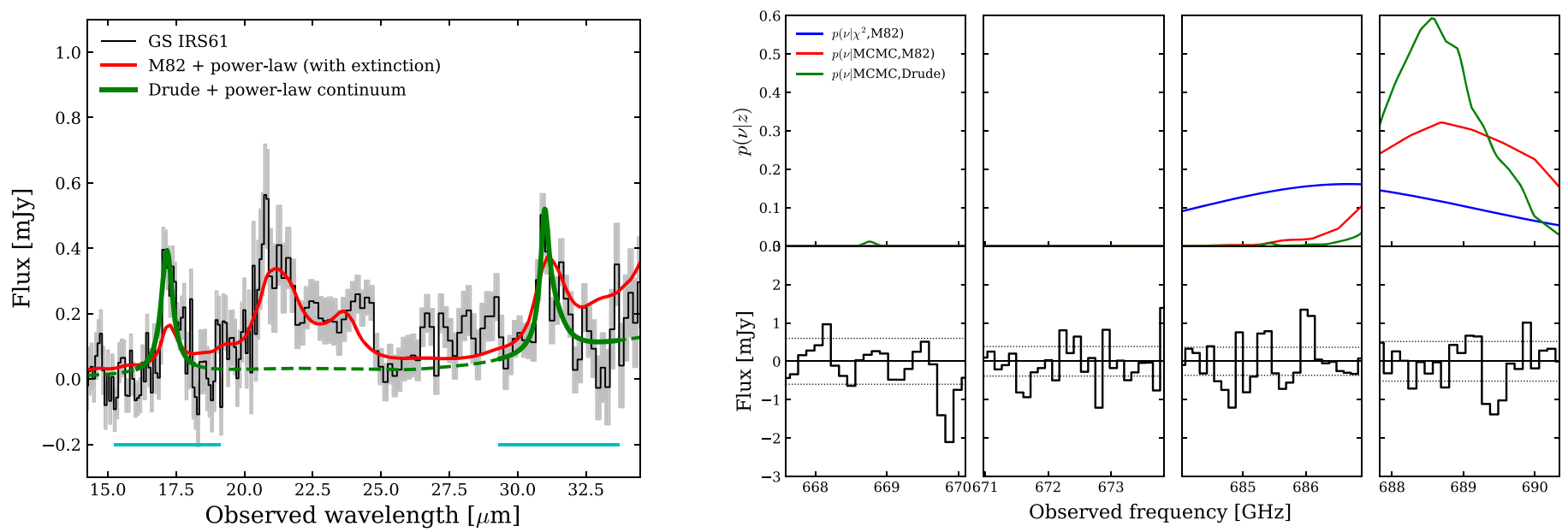

Figure 11. The importance of model selection in estimating redshifts from PAH spectra. Left panel: GS IRS61's Spitzer IRS spectrum. Over-plotted in green is our best-fit Lorentzian model to the 6.2 and $11.3 \mu \mathrm{m}$ PAH complexes. Shown in red is the AGN-SFG decomposition model of Kirkpatrick et al. (2015), which we re-fit to GS IRS61's spectrum using the same MCMC package as was used in fitting the other model. Regions included in the fit are shown with a solid line, whereas dashed lines indicate wavelengths masked from each model. Right panel: the bottom panels show GS IRS61's ALMA Band 9 spectrum, separated into regions of contiguous baseband coverage. The dotted black lines correspond to $\pm 1 \sigma$. The upper panels show $p(\nu \mid z)$, the probability of [C II] being redshift to each observed frequency, for different fitting methods. The red and green lines correspond to the MCMC-derived redshift posteriors from fits in the left panel. The blue line shows a Gaussian approximation of $p(\nu \mid z)$ from $\chi^{2}$ minimization fits with the AGN-SFG decomposition model, originally used to plan the observations. From the green curve, we calculate the probability of having the [C II] line fall in the ALMA bandpass to be 0.93 in GS IRS61.

host to a diverse range of spectral features from rotational lines of molecular hydrogen to bending and stretching modes of PAH molecules. In the low-S/N regime characteristic of highredshift observations, only the brightest PAH features remain distinctly observable. These features are intrinsically broad with intensities $I_{\nu}^{(r)}$ well-fit by Lorentzian (Drude) profiles:

$$
I_{\nu}^{(r)}=\frac{b_{r} \gamma_{r}^{2}}{\left.\left(\lambda / \lambda_{r}-\lambda_{r} / \lambda\right)^{2}+\gamma_{r}^{2}\right)}
$$

where following the convention of Smith et al. (2007), $r$ specifies a given PAH complex with central wavelength $\lambda_{r}$, fractional full width at half maximum (FWHM) $\gamma_{r}$ and central intensity $b_{r}$. Lorentzian profiles are the theoretical spectrum for a classical damped harmonic oscillator, and carry more power in their extended wings compared to a Gaussian. As a result, individual line emission is difficult to separate from adjacent PAH features, as well as any underlying stellar and dust continuum (see Smith et al. 2007 for examples at low-redshift).

Owing to the number of blended line profiles between 5 and $15 \mu \mathrm{m}$, PAH flux densities in this wavelength domain are sensitive to the measurement technique (see Smith et al. 2007 for a thorough analysis). In particular, how the continuum around each PAH feature is estimated can lead to variations in measured line fluxes and equivalent widths by up to a factor of four (Sajina et al. 2007; Smith et al. 2007; Pope et al. 2008). For this reason, we focus our analysis on the 6.2 and $11.3 \mu \mathrm{m}$ PAH luminosities, as these features are comparatively isolated from adjacent lines and trace the total PAH luminosity $\left(L_{\mathrm{PAH}}\right)$ with low scatter in local and high- $z$ star-forming galaxies (Smith et al. 2007; Pope et al. 2008).

Inferring a redshift from $\mathrm{PAH}$ features at low $\mathrm{S} / \mathrm{N}$ and low spectral resolution $(R \sim 100)$ is complicated by the many broad and blended PAH lines. Prior to our ALMA observations, redshifts were determined via the spectral decomposition model of Kirkpatrick et al. (2015), which fits mid-IR spectra with an AGN power-law component, a fixed star-forming galaxy PAH template, and dust extinction. This model works well for separating AGNs and star-formation components (i.e., calculating $f_{\mathrm{AGN}, \mathrm{MIR}}$, Table 1) but does not always reproduce observed PAH intensities as demonstrated in Figure 11 (left panel). Peak emission at line center places the most constraint on a galaxy's systemic redshift. Therefore, we adopt a simpler model of Lorentzian profiles plus a power-law continuum to fit only the 6.2 and $11.3 \mu \mathrm{m}$ PAH complexes. Using this technique, we leverage the relatively isolated lines to measure the target's redshift. In the restframe, our model is

$$
I_{\nu}=N_{\mathrm{pl}} \lambda^{\alpha} e^{-\tau_{\nu, \mathrm{pl}}}+\sum_{r} I_{\nu}^{(r)}\left(b_{r} \mid \lambda_{r}, \gamma_{r}\right) e^{-\tau_{\nu, \mathrm{pl}}}
$$

where $N_{\mathrm{pl}}$ is the power-law scale factor and $\alpha$ is the mid-IR spectral index. We assume a wavelength-dependent Milky Way dust attenuation law for the optical depth parameter $\tau_{\nu, \mathrm{pl}}$ (Weingartner \& Draine 2001). This assumption has minimal to no impact on our results given that the primary purpose of the power-law component is to approximate continuum emission in the vicinity of each $\mathrm{PAH}$ feature. The second term in Equation (4) sums over the various PAH complexes included in the fit, each described by a Lorentzian profile (Equation (3)).

To fit for $b_{r}$ and $z$, we fix the central wavelengths of the 6.2 and $11.3 \mu \mathrm{m}$ PAH features $\left(\lambda_{r}\right)$ and $\gamma_{r}$ to their values derived in Smith et al. (2007). This implicitly assumes comparable dust grain properties between high- $z$ (U)LIRGs and the inner kpc regions of galaxies from the Spitzer Infrared Nearby Galaxies Survey (SINGS; Kennicutt et al. 2003) used to calibrate the PAH free parameters. Many such features observed in low- $z$ star-forming galaxies are also seen in high- $z$ dusty systems, suggesting that the grain properties responsible for the intrinsically brightest PAH complexes (e.g., 6.2, 7.7, 8.6, $11.3 \mu \mathrm{m}$ ) do not change between $z \sim 2$ and today (Pope et al. 2013; Kirkpatrick et al. 2015). Although the $6.2 \mu \mathrm{m}$ peak can shift by $\Delta \lambda_{r} \sim 0.1 \mu \mathrm{m}$ from target to target in the Milky Way, these variations are related to the illumination source and are relatively stable for both individual and averaged $\mathrm{H}$ II regions and PDRs in the Milky Way, which are expected to dominate 
mid-IR emission in star-forming galaxies (Section 2.2.2 of Tielens 2008; van Diedenhoven et al. 2004).

We fit our model (Equation (4)) to each IRS spectrum using the Markov Chain Monte Carlo (MCMC) code emcee, an open-sourced package designed to minimize the number of tunable parameters embedded in a Markov Chain algorithm (Foreman-Mackey et al. 2013). We assume uniform priors on $N_{\mathrm{pl}}, \alpha, \tau_{\nu, \mathrm{pl}}, z$, and $b_{r}$, and restrict the fit to spectral domains around the 6.2 and $11.3 \mu \mathrm{m}$ PAH features (see cyan horizontal lines in Figure 2). The 6.2 and $11.3 \mu \mathrm{m}$ features are unambiguous and readily identified by the code without confusion.

Once the fits have been run, we marginalize over all free parameters and extract from each MCMC chain a redshift posterior probability distribution function $p(z)$. We quote the redshift that maximizes the likelihood function as $z_{\text {IRS }}$, and adopt uncertainties from the minimum and maximum redshifts within the 68th percentile of $p(z)$. Next, we use the local continuum around the 6.2 and $11.3 \mu \mathrm{m}$ line to estimate $L_{6.2 \mu \mathrm{m}}$ and $L_{11.3 \mu \mathrm{m}}$, following measurement methods used in the literature for direct comparison with published values (e.g., Uchida et al. 2000; Peeters et al. 2002; Pope et al. 2008, 2013). Error bars on $L_{6.2 \mu \mathrm{m}}$ and $L_{11.3 \mu \mathrm{m}}$ are derived using Monte Carlo analysis, whereby the observed spectrum is perturbed by pixel noise prior to re-calculating the line-flux and PAH feature luminosity. This process is repeated 1000 times, after which we quote the standard deviation of all iterations as the $1 \sigma$ error. Final measurements and errors of $z_{\mathrm{IRS}}, L_{6.2 \mu \mathrm{m}}$, and $L_{11.3 \mu \mathrm{m}}$ are provided in Table 1. We note that silicate absorption at $9.7 \mu \mathrm{m}$ can potentially impact the $11.3 \mu \mathrm{m}$ PAH feature shape and luminosity. There is little evidence for strong silicate absorption in the spectral decomposition shown in Figure 2; however, the low-S/N data is consistent with optical depths of the $9.7 \mu \mathrm{m}$ feature $\tau_{9.7} \approx 0-2$. At this opacity, the $11.3 \mu \mathrm{m} \mathrm{PAH}$ feature strength is decreased by a factor of 1.4 at most (Smith et al. 2007), which is within the uncertainty of our measure-

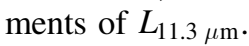

\section{Appendix B \\ Calculating the Probability of Observing [C II] from Total Bandpass Coverage}

In this section, we consider the uncertainties on the redshifts coupled with the ALMA bandpass to calculate the probabilities of observing [C II] for each galaxy in our sample. This analysis is crucial before one can measure upper limits on [C II] from ALMA data containing frequency gaps in baseband coverage. In designing the ALMA Cycle 5 observations, redshifts were determined for each source by fitting a single star-forming PAH template to each galaxy. As demonstrated by Figure 11 (left panel), this method insufficiently matches the brightest PAH emission compared to a Lorentzian profile technique. While both fitting approaches estimate a redshift within $\pm 1 \sigma$ of each other corresponding to $\Delta z=0.014$, on average, differences on the order of $\Delta z \sim 0.01$ can shift the [C II] line in or out of the ALMA spectral windows at the highest frequencies. For this reason, our observations may have missed [C II] in some galaxies.

To quantify $p(l \mid$ ALMA, $\Delta z)$, we take redshift posterior probability distributions from our MCMC fits to the IRS spectra (Section 3.1, Figure 2) and from these, compute $p\left(\nu_{[\mathrm{C} \text { II] }} \mid z\right)$ : the probability [C II] would be redshifted to a given frequency. Next, we integrate $p\left(\nu_{[\mathrm{C} \text { ㅍ] }} \mid z\right)$ first over all frequencies and then over the frequency domain covered by our bandpass tunings, which is typically $\sim 10 \mathrm{GHz}$ between 632 and $687 \mathrm{GHz}$ not counting gaps between individual spectral windows. Thus, we quantitatively derive $p(l \mid$ ALMA, $\Delta z)$ according to the following prescription:

$$
p(l \mid \text { ALMA }, \Delta z) \equiv \frac{\sum_{i} \int_{\min \left(\nu_{i}\right)}^{\max \left(\nu_{i}\right)} p\left(\nu_{[\mathrm{C} \mathrm{II}]} \mid z\right) d \nu}{\int_{-\infty}^{+\infty} p\left(\nu_{[\mathrm{C} \text { II] }} \mid z\right) d \nu}
$$

where the summation treats each ALMA spectral window independently and avoids gaps in wavelength coverage. Figure 11 (right panels) graphically demonstrates this technique for GS IRS61, the only galaxy in our observations where $p(l \mid$ ALMA,$\Delta z)>90 \%$. Estimates of $p(l \mid$ ALMA, $\Delta z)$ for all other targets are given in Table 3.

Additional redshift constraint from restframe optical spectroscopy can be used to improve the estimate of $p(l \mid$ ALMA, $\Delta z)$. In principle, we would multiply optical redshift posteriors with our MCMC-derived $p(z)$ and integrate the product. We checked for optical spectroscopic redshifts by matching to catalogs from 3D-HST grism (Momcheva et al. 2016), VLT/FORS-2 (Vanzella et al. 2008), VANDELS (McLure et al. 2017), MUSE GTO surveys, and ALESS (Danielson et al. 2017). GS IRS50 and GS IRS58 have grism spectroscopic redshifts consistent with $z_{\mathrm{PAH}}$ but with higher uncertainty. GS IRS20 and GS IRS61 have C-grade VLT/ FORS-2 spectra, and grism redshifts completely inconsistent with the PAH features in both galaxies by $\Delta z=0.2-0.3$, greater than 10 times the uncertainty on their PAH-derived redshifts. GS IRS46 and GS IRS52 do not have optical spectra. In summary, no significantly accurate optical spectroscopic redshifts $(\Delta z<0.01)$ consistent with $z_{\mathrm{PAH}}$ were found that changed our results using only PAH fits.

\section{ORCID iDs}

Jed McKinney (1) https://orcid.org/0000-0002-6149-8178 Alexandra Pope (i) https://orcid.org/0000-0001-8592-2706 Lee Armus (i) https://orcid.org/0000-0003-3498-2973

Ranga-Ram Chary (1) https://orcid.org/0000-0001-7583-0621 Tanio Díaz-Santos (1) https://orcid.org/0000-0003-0699-6083 Allison Kirkpatrick ㄴ https://orcid.org/0000-0002-1306-1545

\section{References}

Armus, L., Charmandaris, V., Bernard-Salas, J., et al. 2007, ApJ, 656, 148 Armus, L., Mazzarella, J. M., Evans, A. S., et al. 2009, PASP, 121, 559 Bakes, E. L. O., \& Tielens, A. G. G. M. 1994, ApJ, 427, 822 Brandl, B. R., Bernard-Salas, J., Spoon, H. W. W., et al. 2006, ApJ, 653, 1129 Brisbin, D., Ferkinhoff, C., Nikola, T., et al. 2015, ApJ, 799, 13 Bussmann, R. S., Pérez-Fournon, I., Amber, S., et al. 2013, ApJ, 779, 25 Calistro Rivera, G., Hodge, J. A., Smail, I., et al. 2018, ApJ, 863, 56 Capak, P. L., Carilli, C., Jones, G., et al. 2015, Natur, 522, 455 Carilli, C. L., \& Walter, F. 2013, ARA\&A, 51, 105

Carniani, S., Maiolino, R., Pallottini, A., et al. 2017, A\&A, 605, A42 Carniani, S., Maiolino, R., Smit, R., \& Amorín, R. 2018, ApJL, 854, L7 Chary, R., \& Elbaz, D. 2001, ApJ, 556, 562

Cormier, D., Abel, N. P., Hony, S., et al. 2019, A\&A, 626, A23 Cortzen, I., Garrett, J., Magdis, G., et al. 2019, MNRAS, 482, 1618 Croxall, K. V., Smith, J. D., Pellegrini, E., et al. 2017, ApJ, 845, 96 Croxall, K. V., Smith, J. D., Wolfire, M. G., et al. 2012, ApJ, 747, 81 Dale, D. A., Smith, J. D. T., Schlawin, E. A., et al. 2009, ApJ, 693, 1821 Danielson, A. L. R., Swinbank, A. M., Smail, I., et al. 2017, ApJ, 840, 78 De Breuck, C., Williams, R. J., Swinbank, M., et al. 2014, Msngr, 156, 38 Decarli, R., Walter, F., Venemans, B. P., et al. 2018, ApJ, 854, 97 Díaz-Santos, T., Armus, L., Charmandaris, V., et al. 2013, ApJ, 774, 68 Díaz-Santos, T., Armus, L., Charmandaris, V., et al. 2014, ApJL, 788, L17 
Díaz-Santos, T., Armus, L., Charmandaris, V., et al. 2017, ApJ, 846, 32 Elbaz, D., Leiton, R., Nagar, N., et al. 2018, A\&A, 616, A110 Fadda, D., Yan, L., Lagache, G., et al. 2010, ApJ, 719, 425

Foreman-Mackey, D., Hogg, D. W., Lang, D., \& Goodman, J. 2013, PASP 125,306

Gallerani, S., Neri, R., Maiolino, R., et al. 2012, A\&A, 543, A114

Genzel, R., Burkert, A., Bouché, N., et al. 2008, ApJ, 687, 59

Genzel, R., Tacconi, L. J., Lutz, D., et al. 2015, ApJ, 800, 20

Giavalisco, M., Ferguson, H. C., Koekemoer, A. M., et al. 2004, ApJL, 600, L93

Goldsmith, P. F., Langer, W. D., Pineda, J. L., \& Velusamy, T. 2012, ApJS, 203, 13

Graciá-Carpio, J., Sturm, E., Hailey-Dunsheath, S., et al. 2011, ApJL, 728, L7

Grogin, N. A., Kocevski, D. D., Faber, S. M., et al. 2011, ApJS, 197, 35

Gullberg, B., De Breuck, C., Vieira, J. D., et al. 2015, MNRAS, 449, 2883

Gullberg, B., Swinbank, A. M., Smail, I., et al. 2018, ApJ, 859, 12

Hashimoto, T., Inoue, A. K., Mawatari, K., et al. 2019, PASJ, 71, 71

Hashimoto, T., Inoue, A. K., Tamura, Y., et al. 2018, arXiv:1811.00030

Helou, G., Malhotra, S., Hollenbach, D. J., Dale, D. A., \& Contursi, A. 2001, ApJL, 548, L73

Houck, J. R., Soifer, B. T., Weedman, D., et al. 2005, ApJL, 622, L105

Ibar, E., Lara-López, M. A., Herrera-Camus, R., et al. 2015, MNRAS, 449, 2498

Ivison, R. J., Swinbank, A. M., Swinyard, B., et al. 2010, A\&A, 518, L35

Jones, G. C., Willott, C. J., Carilli, C. L., et al. 2017, ApJ, 845, 175

Kartaltepe, J. S., Mozena, M., Kocevski, D., et al. 2015, ApJS, 221, 11

Kaufman, M. J., Wolfire, M. G., Hollenbach, D. J., \& Luhman, M. L. 1999, ApJ, 527, 795

Kennicutt, R. C., Jr., Armus, L., Bendo, G., et al. 2003, PASP, 115, 928

Kennicutt, R. C. J., Jr. 1998, ARA\&A, 36, 189

Kereš, D., Katz, N., Fardal, M., Davé, R., \& Weinberg, D. H. 2009, MNRAS, 395,160

Kereš, D., Katz, N., Weinberg, D. H., \& Davé, R. 2005, MNRAS, 363, 2

Kirkpatrick, A., Pope, A., Alexander, D. M., et al. 2012, ApJ, 759, 139

Kirkpatrick, A., Pope, A., Sajina, A., et al. 2015, ApJ, 814, 9

Koekemoer, A. M., Faber, S. M., Ferguson, H. C., et al. 2011, ApJS, 197, 36

Lang, P., Schinnerer, E., Smail, I., et al. 2019, arXiv:1905.06960

Laurent, O., Mirabel, I. F., Charmandaris, V., et al. 2000, A\&A, 359, 887

Le Fèvre, O., Béthermin, M., Faisst, A., et al. 2019, arXiv:1910.09517

Lilly, S. J., Le Fevre, O., Hammer, F., \& Crampton, D. 1996, ApJL, 460, L1

Liu, D., Schinnerer, E., Groves, B., et al. 2019, arXiv:1910.12883

Luhman, M. L., Satyapal, S., Fischer, J., et al. 1998, ApJL, 504, L11

Luhman, M. L., Satyapal, S., Fischer, J., et al. 2003, ApJ, 594, 758

Madau, P., \& Dickinson, M. 2014, ARA\&A, 52, 415

Madau, P., Ferguson, H. C., Dickinson, M. E., et al. 1996, MNRAS, 283, 1388

Magdis, G. E., Rigopoulou, D., Hopwood, R., et al. 2014, ApJ, 796, 63

Maiolino, R., Carniani, S., Fontana, A., et al. 2015, MNRAS, 452, 54

Malhotra, S., Helou, G., Stacey, G., et al. 1997, ApJL, 491, L27

Malhotra, S., Kaufman, M. J., Hollenbach, D., et al. 2001, ApJ, 561, 766

Marshall, J. A., Herter, T. L., Armus, L., et al. 2007, ApJ, 670, 129

Matthee, J., Sobral, D., Boone, F., et al. 2017, ApJ, 851, 145

McLure, R., Pentericci, L. \& VANDELS Team 2017, Msngr, 167, 31

McMullin, J. P., Waters, B., Schiebel, D., Young, W., \& Golap, K. 2007, in ASP Conf. Ser. 376, Astronomical Data Analysis Software and Systems XVI, ed. R. A. Shaw, F. Hill, \& D. J. Bell (San Francisco, CA: ASP), 127
Momcheva, I. G., Brammer, G. B., van Dokkum, P. G., et al. 2016, ApJS, 225, 27

Muñoz, J. A., \& Oh, S. P. 2016, MNRAS, 463, 2085

Nguyen, H. T., Schulz, B., Levenson, L., et al. 2010, A\&A, 518, L5

Oteo, I., Ivison, R. J., Dunne, L., et al. 2016, ApJ, 827, 34

Peeters, E., Hony, S., Van Kerckhoven, C., et al. 2002, A\&A, 390, 1089

Pentericci, L., Carniani, S., Castellano, M., et al. 2016, ApJL, 829, L11

Pope, A., Chary, R.-R., Alexander, D. M., et al. 2008, ApJ, 675, 1171

Pope, A., Wagg, J., Frayer, D., et al. 2013, ApJ, 772, 92

Rawle, T. D., Egami, E., Bussmann, R. S., et al. 2014, ApJ, 783, 59

Riechers, D. A., Bradford, C. M., Clements, D. L., et al. 2013, Natur, 496, 329

Rigopoulou, D., Hopwood, R., Magdis, G. E., et al. 2014, ApJL, 781, L15

Rybak, M., Calistro Rivera, G., Hodge, J. A., et al. 2019, arXiv:1901.10027

Sajina, A., Yan, L., Armus, L., et al. 2007, ApJ, 664, 713

Sajina, A., Yan, L., Lutz, D., et al. 2008, ApJ, 683, 659

Sanders, R. L., Shapley, A. E., Kriek, M., et al. 2015, ApJ, 799, 138

Sargsyan, L., Samsonyan, A., Lebouteiller, V., et al. 2014, ApJ, 790, 15

Schaerer, D., Boone, F., Jones, T., et al. 2015, A\&A, 576, L2

Scoville, N., Lee, N., Vanden Bout, P., et al. 2017, ApJ, 837, 150

Shipley, H. V., Papovich, C., Rieke, G. H., Brown, M. J. I., \& Moustakas, J. 2016, ApJ, 818, 60

Shivaei, I., Reddy, N. A., Shapley, A. E., et al. 2017, ApJ, 837, 157

Smit, R., Bouwens, R. J., Carniani, S., et al. 2018, Natur, 553, 178

Smith, J. D. T., Croxall, K., Draine, B., et al. 2017, ApJ, 834, 5

Smith, J. D. T., Draine, B. T., Dale, D. A., et al. 2007, ApJ, 656, 770

Speagle, J. S., Steinhardt, C. L., Capak, P. L., \& Silverman, J. D. 2014, ApJS, 214,15

Stacey, G. J., Hailey-Dunsheath, S., Ferkinhoff, C., et al. 2010, ApJ, 724, 957

Stierwalt, S., Armus, L., Charmandaris, V., et al. 2014, ApJ, 790, 124

Stierwalt, S., Armus, L., Surace, J. A., et al. 2013, ApJS, 206, 1

Sturm, E., Lutz, D., Tran, D., et al. 2000, A\&A, 358, 481

Sutter, J., Dale, D. A., Croxall, K. V., et al. 2019, ApJ, 886, 60

Tacconi, L. J., Genzel, R., Neri, R., et al. 2010, Natur, 463, 781

Tacconi, L. J., Genzel, R., Saintonge, A., et al. 2018, ApJ, 853, 179

Tacconi, L. J., Neri, R., Genzel, R., et al. 2013, ApJ, 768, 74

Tadaki, K.-i., Iono, D., Hatsukade, B., et al. 2019, ApJ, 876, 1

Tielens, A. G. G. M. 2008, ARA\&A, 46, 289

Tielens, A. G. G. M., \& Hollenbach, D. 1985, ApJ, 291, 722

Tran, Q. D., Lutz, D., Genzel, R., et al. 2001, ApJ, 552, 527

Uchida, K. I., Sellgren, K., Werner, M. W., \& Houdashelt, M. L. 2000, ApJ, 530,817

Valtchanov, I., Virdee, J., Ivison, R. J., et al. 2011, MNRAS, 415, 3473

van Diedenhoven, B., Peeters, E., Van Kerckhoven, C., et al. 2004, ApJ, 611,928

Vanzella, E., Cristiani, S., Dickinson, M., et al. 2008, A\&A, 478, 83

Veilleux, S., Rupke, D. S. N., Kim, D. C., et al. 2009, ApJS, 182, 628

Walter, F., Decarli, R., Carilli, C., et al. 2012, Natur, 486, 233

Watson, W. D., \& Salpeter, E. E. 1972, ApJ, 174, 321

Weingartner, J. C., \& Draine, B. T. 2001, ApJ, 548, 296

Wolfire, M. G., Tielens, A. G. G. M., \& Hollenbach, D. 1990, ApJ, 358, 116

Wu, Y., Helou, G., Armus, L., et al. 2010, ApJ, 723, 895

Yan, L., Chary, R., Armus, L., et al. 2005, ApJ, 628, 604

Zanella, A., Daddi, E., Magdis, G., et al. 2018, MNRAS, 481, 1976 